\title{
Quantitative proteomics of acutely-isolated mouse microglia identifies novel immune Alzheimer's disease-related proteins
}

Srikant Rangaraju ${ }^{1 *} \mathbb{D}_{\text {, Eric B. Dammer }}^{2 \dagger}$, Syed Ali Raza', Tianwen Gao ${ }^{1}$, Hailian Xiao ${ }^{1}$, Ranjita Betarbet ${ }^{1}$, Duc M. Duong ${ }^{2}$, James A. Webster ${ }^{1}$, Chadwick M. Hales ${ }^{1}$, James J. Lah ${ }^{1}$, Allan I. Levey ${ }^{1}$ and Nicholas T. Seyfried ${ }^{1,2^{*}}$

\begin{abstract}
Background: Microglia are innate immune cells of the brain that perform phagocytic and inflammatory functions in disease conditions. Transcriptomic studies of acutely-isolated microglia have provided novel insights into their molecular and functional diversity in homeostatic and neurodegenerative disease states. State-of-the-art mass spectrometry methods can comprehensively characterize proteomic alterations in microglia in neurodegenerative disorders, potentially providing novel functionally relevant molecular insights that are not provided by transcriptomics. However, comprehensive proteomic profiling of adult primary microglia in neurodegenerative disease conditions has not been performed.
\end{abstract}

Methods: We performed quantitative mass spectrometry based proteomic analyses of purified CD11 $\mathrm{b}^{+}$acutelyisolated microglia from adult (6 mo) mice in normal, acute neuroinflammatory (LPS-treatment) and chronic neurodegenerative states (5xFAD model of Alzheimer's disease [AD]). Differential expression analyses were performed to characterize specific microglial proteomic changes in 5xFAD mice and identify overlap with LPSinduced pro-inflammatory changes. Our results were also contrasted with existing proteomic data from wild-type mouse microglia and from existing microglial transcriptomic data from wild-type and 5xFAD mice. Neuropathological validation studies of select proteins were performed in human AD and 5xFAD brains.

Results: Of 4133 proteins identified, 187 microglial proteins were differentially expressed in the 5xFAD mouse model of AD pathology, including proteins with previously known (Apoe, Clu and Htra1) as well as previously unreported relevance to AD biology (Cotl1 and Hexb). Proteins upregulated in 5xFAD microglia shared significant overlap with pro-inflammatory changes observed in LPS-treated mice. Several proteins increased in human AD brain were also upregulated by 5xFAD microglia (Aß peptide, Apoe, Htra1, Cotl1 and Clu). Cotl1 was identified as a novel microglia-specific marker with increased expression and strong association with AD neuropathology. Apoe protein was also detected within plaque-associated microglia in which Apoe and A $\beta$ were highly co-localized, suggesting a role for Apoe in phagocytic clearance of $A \beta$.

Conclusions: We report a comprehensive proteomic study of adult mouse microglia derived from acute neuroinflammation and AD models, representing a valuable resource to the neuroscience research community. We highlight shared and unique microglial proteomic changes in acute neuroinflammation aging and AD mouse models and identify novel roles for microglial proteins in human neurodegeneration.

Keywords: Microglia, Neurodegeneration, Neuroinflammation, Immunology, Proteomics, Mass spectrometry, Alzheimer's disease

\footnotetext{
*Correspondence: Srikant.rangaraju@emory.edu; ntseyfri@emory.edu

'Srikant Rangaraju and Eric B. Dammer contributed equally to this work.

${ }^{1}$ Department of Neurology, Emory University, Atlanta, GA 30322, USA

Full list of author information is available at the end of the article
}

(c) The Author(s). 2018 Open Access This article is distributed under the terms of the Creative Commons Attribution 4.0 International License (http://creativecommons.org/licenses/by/4.0/), which permits unrestricted use, distribution, and reproduction in any medium, provided you give appropriate credit to the original author(s) and the source, provide a link to the Creative Commons license, and indicate if changes were made. The Creative Commons Public Domain Dedication waiver (http://creativecommons.org/publicdomain/zero/1.0/) applies to the data made available in this article, unless otherwise stated. 


\section{Background}

Microglia are the primary innate immune cells of the central nervous system (CNS). These yolk sac-derived myeloid cells take up residence in the CNS during embryogenesis [1]. Microglia perform both homeostatic functions such as synaptic pruning and environmental sensing and under disease states, can adopt transcriptionally distinct profiles that can mediate both neurotoxic as well as neuroprotective and phagocytic roles [2-4]. In aging and in chronic neurodegenerative diseases such as Alzheimer's disease (AD), microglia adopt unique disease-associated-microglial (DAM) transcriptional profiles and it is suspected that DAM can mediate phagocytic clearance of amyloid beta $(\mathrm{A} \beta)$, a process that becomes progressively dysregulated and ineffective with disease progression [5, 6]. Although transcriptional profiling provides an in-depth understanding of gene-expression changes in disease, modest agreement between transcriptional level changes and protein level changes have been observed across various studies and cell types [7, 8]. Since functional phenotypes are largely mediated by protein effectors, proteomic studies should provide an important perspective for understanding pathophysiological changes that result in neurodegeneration. Recent advances in mass spectrometry methods have made deep proteomic profiling of relatively smaller numbers of cells feasible [9].

The proteome of microglia from young and aging wild-type (WT) mice has been described $[8,10]$, and more recently, a limited proteomic analysis of acutely-isolated microglia from the 5xFAD mouse model of AD pathology identified early pro-inflammatory microglial activation prior to $\mathrm{A} \beta$ deposition [11]. However, comprehensive and comparative proteomic studies of adult microglia under homeostatic conditions, acute neuroinflammatory and chronic neurodegenerative disease states have not been reported. We report the deepest quantitative and most comprehensive proteome of acutely isolated microglia derived from adult mice and from mouse models of acute neuroinflammation and AD pathology. We report unexpected similarities between murine models of acute neuroinflammation and chronic neurodegeneration, in addition to highlighting unique neurodegeneration-specific immune changes seen in 5xFAD mice. We also highlight the complementary nature of transcriptomic and proteomic data in understanding the dynamic nature of microglial gene and protein expression changes under different in-vivo conditions. Furthermore, we identify unappreciated microglia-specific markers relevant to human $\mathrm{AD}$ as and suggest novel AD-relevant biology for proteins that were not thought to be expressed by microglia. Therefore, this microglial proteome represents a valuable resource to the neuroimmunology and neurodegenerative disease research communities, serving as a reference for future comprehensive immune profiling and target validation studies.

\section{Methods \\ Animals}

Female C57BL/6 J and female 5xFAD mice (on a C57BL/6 J background) used for the studies were housed in the Department of Animal Resources at Emory University under standard conditions. Institutional Animal Care and Use Committee approval was obtained prior to in-vivo work and all work was performed in strict accordance with the Guide for the Care and Use of Laboratory Animals of the National Institutes of Health. Adult mice were given intraperitoneal LPS injections $(10 \mu \mathrm{g} /$ dose $\times 4$ daily doses $)$ to induce acute neuroinflammation $[12,13]$. If $\geq 25 \%$ of weight loss was observed, animals were euthanized. Three groups of adult 6-7 mo old C57BL/6 J mice ( $n=3$ pools each, 2 mouse brains per pool) were used for our studies. Wild-type (WT) mice (untreated), WT mice treated with LPS for 4 consecutive days (IP, $20 \mu \mathrm{g} /$ dose) and 5xFAD mice were euthanized followed by cardiac perfusion as previously described [13].

\section{Acute isolation and enrichment of CD11 $\mathrm{b}^{+}$microglia and brain mononuclear phagocytes}

The brain was dissected and brain mononuclear cells were isolated as previously described by Percoll gradient centrifugation [13]. Mechanical homogenization of the brain was performed rather than enzymatic digestion due to two reasons: i) in our experience, live microglial cell yield using enzymatic or mechanical dissociation methods are very similar $(\approx 200,000$ mononuclear cells per brain), and ii) enzymatic digestion may cleave cell surface proteins, thereby potentially decreasing the likelihood of identifying cell surface proteins by mass spectrometry. Isolated cells were then further purified by CD11b positive selection using a MACS column (Miltenyi Biotec Cat\#130-093-636) to selectively enrich microglia and brain mononuclear phagocytes. Positive selection enriched the $\mathrm{CD}_{11 b^{+}}$population from 60 to $95 \%$ as confirmed by flow cytometry. On average, 200,000 live $\mathrm{CD}_{11 b^{+}}$cells were isolated from each brain. Whole cell microglial lysates were prepared and processed for analysis.

\section{Sample preparation for mass spectrometry analysis}

Cell pellets were flash frozen and stored at $-80{ }^{\circ} \mathrm{C}$ until ready for protein extraction. Cells were lysed in $8 \mathrm{M}$ urea lysis buffer (8 M urea, $100 \mathrm{mM}$ NaHPO4, pH 8.5) with HALT protease and phosphatase inhibitor cocktail (ThermoFisher). Each sample was then sonicated for 3 cycles consisting of $5 \mathrm{~s}$ of active sonication at $30 \%$ amplitude followed by $15 \mathrm{~s}$ on ice. Samples were then 
centrifuged for $5 \mathrm{~min}$ at $15,000 \mathrm{~g}$ and the supernatant was transferred to a new tube. Protein concentration was determined by bicinchoninic acid (BCA) assay (Pierce) and $20 \mu \mathrm{g}$ of each sample was aliquoted.

Protein digestion and sample cleanup was performed as previously published [14]. Briefly, protein samples were reduced with $1 \mathrm{mM}$ dithiothreitol (DTT) for 30 mins, alkylated with $5 \mathrm{mM}$ iodoacetamide (IAA) in the dark for another 30 mins and then 8-fold diluted with $50 \mathrm{mM}$ triethylammonium bicarbonate (TEAB). Overnight digestion was performed with 1:100 (w/w) Lysyl endopeptidase (Wako) followed by an additional 12-h digestion with Trypsin at 1:50 (w/w). The peptide solutions were acidified, and desalted with a $\mathrm{C}_{18}$ Sep-Pak column (Waters). A $2 \mu \mathrm{g}$ equivalent of each sample elution was pooled and used to create a global internal standard (GIS) and all samples were dried under vacuum.

Tandem mass tag (TMT) peptide labeling was performed according to manufacturer's instructions and as previously described [14]. One batch of 10-plex TMT kits (Thermo Fisher) was used to label the 9 samples and one GIS mixtures. In this batch, TMT channel 131 was used to label the GIS standard, while the 9 remaining TMT channels were used to label each individual sample. Electrostatic repulsion-hydrophilic interaction chromatography (ERLIC) offline fractionation was performed as previously described $[14,15]$. Briefly, dried samples were re-suspended in $100 \mu \mathrm{L}$ of ERLIC buffer A ( $90 \%$ acetonitrile with $0.1 \%$ acetic acid) and separated on a PolyWAX LP column $(20 \mathrm{~cm}$ by $3.2 \mathrm{~mm}$ packed with $300 \AA 5 \mu \mathrm{m}$ beads (PolyLC Inc.) and elution fractions were recovered over a 45 -min gradient from 0 to $50 \%$ ERLIC buffer B ( $30 \%$ ACN with $0.1 \%$ FA). The original 44 fractions were combined as previously described into 21 and dried under vacuum $[14,15]$.

\section{Mass spectrometry analysis and TMT data acquisition}

Assuming equal distribution of peptide concentration across all ERLIC fractions, $10 \mu \mathrm{L}$ of loading buffer $(0.1 \%$ TFA) was added to each of the fractions and $2 \mu \mathrm{L}$ was separated on a $25 \mathrm{~cm}$ long by $75 \mu \mathrm{m}$ internal diameter fused silica column (New Objective, Woburn, MA) packed in-house with $1.9 \mu \mathrm{m}$ Reprosil-Pur $\mathrm{C}_{18}$-AQ resin. The LC-MS/MS platform consisted of a Dionex RSLCnano UPLC coupled to an Orbitrap Fusion mass spectrometer with a Flex nano-electrospray ion source (Thermo Fisher). Sample elution was performed over a gradient of 3 to $30 \%$ Buffer B (0.1\% formic acid in ACN) over $105 \mathrm{mins}$ (flow rate started at $300 \mathrm{nl} / \mathrm{min}$ and ended at $350 \mathrm{nl} / \mathrm{min}$ ), from 30 to $60 \% \mathrm{~B}$ over $20 \mathrm{mins}$ at $350 \mathrm{nl} / \mathrm{min}$, and from 60 to $99 \% \mathrm{~B}$ over $5 \mathrm{mins}$ at $350 \mathrm{nl} / \mathrm{min}$. The column was equilibrated with $1 \% \mathrm{~B}$ for $10 \mathrm{~min}$ at a flow rate that increased from $350 \mathrm{nl} / \mathrm{min}$ to $400 \mathrm{nl} / \mathrm{min}$. The MS was operated in positive ion mode and utilized the synchronous precursor selection (SPS)-MS3 method for reporter ion quantitation as described [14]. The full scan range was $380-1500 \mathrm{~m} / \mathrm{z}$ at a nominal resolution of 120,000 at $200 \mathrm{~m} / \mathrm{z}$ and automatic gain control (AGC) set to $2 \times 10^{5}$. Collision-induced dissociation (CID)-Tandem MS/MS at 35\% normalized collision energy (CE) and higher energy collision dissociation (HCD) SPS-MS3 at 65\% normalized collision energy (CE) were collected at top speed with $3 \mathrm{~s}$ cycles. For SPS, the top 10 product ions were notched and fragmented.

\section{Protein identification and quantification}

Raw data files from Orbitrap Fusion were processed using Proteome Discover (version 2.1). Collected MS/ MS spectra were searched against the UniProt mouse proteome database (54,489 total sequences). SEQUEST parameters were specified as: trypsin enzyme, two missed cleavages allowed, minimum peptide length of 6, TMT tags on lysine residues and peptide $\mathrm{N}$-termini $(+229.162932 \mathrm{Da})$ and carbamidomethylation of cysteine residues $(+57.02146 \mathrm{Da})$ as fixed modifications, oxidation of methionine residues $(+15.99492 \mathrm{Da})$ and deamidation of asparagine and glutamine $(+0.984 \mathrm{Da})$ as a variable modification, precursor mass tolerance of $20 \mathrm{ppm}$, and a fragment mass tolerance of 0.6 Da. Peptide spectral match (PSM) error rates were determined using the target-decoy strategy coupled to Percolator [16] modeling of true and false matches. Reporter ions were quantified from MS3 scans using an integration tolerance of $20 \mathrm{ppm}$ with the most confident centroid setting. An MS2 spectral assignment false discovery rate (FDR) of less than $1 \%$ was achieved by applying the target-decoy strategy. Following spectral assignment, peptides were assembled into proteins and were further filtered based on the combined probabilities of their constituent peptides to a final FDR of $1 \%$. In cases of redundancy, shared peptides were assigned to the protein sequence with the most matching peptides, thus adhering to principles of parsimony. The search results and TMT quantification as well as raw LC-MS/MS files are included in the ProteomeXchange online repository with identifier PXD009137. Even though TMT labeling limits missing values, normalized abundances were zero for some channels in the dataset, up to 3 out of the 9 channels representing individual samples. Missing values were imputed according to the informative missing-ness assumption, such that these values fit into the left tail of the population-wide Gaussian distribution, with a mean 1.8 population-wide standard deviations less than the mean for non-missing data, and randomness of the imputed population was allowed to vary within \pm 0.3 -fold of the same standard deviation. 
Gene ontology enrichment analysis and visualization, pathway analysis and identification of upstream transcriptional regulators

Functional enrichment of the differentially expressed proteins was determined using the GO-Elite (v1.2.5) python package [17]. The set of total proteins identified and quantified $(n=4133)$ was used as the background. Input lists included proteins significantly differentially expressed ( $p<0.05$ by Student's t test) comparing either $5 x$ FAD vs WT or LPS-treated WT vs untreated WT mouse microglial proteomic data. Z-score determines over-representation of ontologies and Fisher's exact test $p$-value was used to assess the significance of the Z-score. GO Elite v1.2.5 command line application with $\mathrm{Z}$ score cutoff of 1.96 and minimum 5 genes per ontology identified ontology enrichments for LPS or AD model-affected proteins, similar to our previously published method [18]. Enrichment Map v2.1.0 Cytoscape plugin [19] was called from Cytoscape v3.5.1 on DAVID-formatted GO-Elite pruned Z-Score output tables. Edge connectivity (Jaccard similarity metric) ranges from 0 (white) to 1 (dark red or blue). Ontology network node colors represent the degree of average $\log _{2}$ fold change for all differentially expressed members of the ontology in the AD-WT and LPS-WT/CT networks. Since no overlap of gene symbols occurs between differential expression list-derived up and down subnetworks, respectively red and blue subnetworks were overlaid to produce the visualizations shown. Differentially expressed protein lists were also used for bioinformatics analysis (MetaCore software, Thomson Reuters) including pathway analyses to identify over-represented molecular and metabolic pathways as well to identify potential upstream transcriptional regulators as previously described [20].

\section{Immunofluorescence microscopy}

For mouse tissue, brains were harvested after cardiac perfusion with ice cold saline, followed by $4 \%$ paraformaldehyde. After overnight fixation, brains were transferred to $30 \%$ sucrose for $24 \mathrm{~h}$ after which $30 \mu \mathrm{m}$ sections were obtained using a cryostat. For all immunohistochemistry studies, three sagittal fixed brain sections from wild-type and 5xFAD mice from equivalent regions were placed on each slide to control for any heterogeneity in staining. For $A \beta$ retrieval, sections were treated with $70 \%$ formic acid for $10 \mathrm{~min}$, washed in buffer, blocked with $3 \%$ hydrogen peroxide and $10 \mu \mathrm{g} / \mathrm{ml}$ of Avidin for $30 \mathrm{~min}$ and then blocked in 10\% normal horse serum prepared in Tris-buffered saline (TBS) for 30 min followed by overnight incubation with primary antibody (anti-Aß42 4G8 antibody [Signet, Cat \# 922002] 1:1000, anti-Apoe antibody [Meridian Life Science, Cat \# K74180B] 1:100 [21], anti-Iba1 antibody [Abcam ab178846], anti-GFAP [Millipore Sigma, Cat \# MAB360] 1:100, anti-CD68 [Abcam ab955] 1:100). Sections were rinsed in TBS and then incubated in the appropriate fluorophore-conjugated secondary antibody (1:500) for 30 min after which tissues were mounted on slides, dried and mounted with mounting medium containing DAPI for nuclear staining (Fluoreshield, Sigma-Aldrich F6057). Confocal microscopy was performed on Leica SP8 multi photon microscope and all image processing was performed using Imaris software. For co-localization analyses, appropriate thresholds for each channel were determined using appropriate negative controls. Immunofluorescence microscopy was performed on an immunofluorescence microscope (Microscope: Olympus BX51 and camera: Olympus DP70) and image processing was performed using ImageJ.

\section{Immunohistochemistry (IHC)}

Cryopreserved pathology-confirmed $\mathrm{AD}(n=3)$ and ageand sex-matched non-disease control $(\mathrm{n}=3)$ frontal cortex tissues were obtained from the Emory Neuropathological core and sectioned using a cryotostat $(30 \mu \mathrm{m}$ slice thickness). Endogenous peroxidase was quenched with $0.3 \%$ hydrogen peroxide for $30 \mathrm{~min}$ and then the sample was blocked with $10 \%$ normal goat serum $/ 0.4 \%$ Triton X-100/Tris-buffered saline (TBS) for $1 \mathrm{~h}$, followed by avidin/biotin binding (Vector labs SP2001) per manufacturer's instructions, followed by overnight incubation with primary antibodies (anti-Cotl1 [Sigma-Aldrich HPA008918, also validated in the Human Protein Atlas] rabbit polyclonal Ab 1:100) [22]. Sections were rinsed with TBS and incubated with appropriate biotin-conjugated secondary antibody followed by Vectastain Elite $A B C$ and diaminobenzidine (DAB) as per manufacturer's recommendations. To exclude nonspecific staining unrelated to polyclonal and monoclonal antibodies, immunostaining was performed with omission of the antibodies but with all other procedures unchanged. Slides were lastly counterstained with hematoxylin. Light microscopy was performed with an Olympus light microscope (Olympus, Center Valley, $\mathrm{PA})$. Images acquired from $4 \mathrm{AD}$ cases and 4 non- $\mathrm{AD} /$ non-disease post-mortem cases (frontal cortex) were used for quantitative analysis to compare Cotl1 immunoreactivity (ImageJ software) as described [23].

\section{Existing proteomic and transcriptomic datasets used for comparative analyses Primary microglial proteomics data}

An existing proteome of acutely isolated and purified microglia as well as other brain cell types (neurons, astrocytes and oligodendroglia) [8] was used as a reference proteome. Although mice used for our study were significantly older (6-7 mo) compared to this previously 
published work (8 weeks), we used this existing proteome as a reference to identify microglial-specific proteins as well as the most abundant proteins identified in microglia. We also referenced this dataset to serve as a background of all identifiable proteins in mouse brain.

\section{Purified microglial transcriptomics}

$\mathrm{CD}_{11 \mathrm{~b}^{+}}$microglia microarray datasets from wild-type and 5xFAD mice were obtained from GEO dataset GSE65067 [24]. Existing RNAseq datasets comparing wild-type to LPS-treated mice were also obtained [25]. Differentially expressed genes were contrasted with observed differentially expressed proteins comparing wild-type to LPS-treated wild-type microglia as well as comparing wild-type vs. 5xFAD microglia.

\section{Human post-mortem brain proteomics data}

Protein expression data were obtained from ProteomeXchange (http://proteomecentral.proteomexchange.org/ cgi/GetDataset?ID=PXD007160) [14]. In this study, TMT mass spectrometry was used to identify 10,230 proteins across AD, Parkinson's disease and non-disease control post-mortem brain (frontal cortex) tissues. Protein expression data from $\mathrm{AD}(n=10)$ and non-disease controls $(n=10)$ were used for differential expression analyses, which were then used for comparison with the current mouse microglial proteome.

\section{Other statistical considerations}

Graphpad Prism (Ver. 5), Microsoft Excel and SPSS (Ver. 22) were used to create graphs and perform statistical analyses. All data are shown as mean \pm SEM. Pairwise two-tailed $\mathrm{t}$-tests were performed in unadjusted analyses comparing groups. Statistical significance was set at $p$ value $\leq 0.05$ for all experiments unless specified separately.

\section{Results}

Quantitative proteomics of acutely isolated adult mouse microglia reveals a highly metabolically active phenotype To describe the proteome of adult mouse microglia, we analyzed whole cell lysates of acutely isolated $C D 11 b^{+}$ MACS-purified microglia isolated from brains of 6-7 mo old wild-type (WT) mice treated with saline or intra-peritoneal LPS for 4 days to induce neuroinflammation [13], as well as from $5 x F A D$ mice (Fig. 1a, 2 brains pooled per sample, $n=3$ samples per group) [26]. MACS purification resulted in $>95 \%$ enrichment of $\mathrm{CD}_{11 b^{+}}$CNS immune cells (Fig. 1b). Among CD11b-enriched cells, CD45 $5^{\text {high }}$ mononuclear phagocytes accounted for only $1.3,2.1$ and $4.1 \%$ cells in untreated WT, LPS-treated WT and 5xFAD mice respectively (Fig. 1c) confirming minimal contamination by CNS-infiltrating macrophages. Microglial whole-cell lysates in $8 \mathrm{M}$ urea buffer were digested by LysC and trypsin, followed by labeling of peptides with TMT reagents, admixture of the TMT 10-plex peptides, followed identification and synchronous precursor selection based MS3 (SPS-MS3) mass spectrometry reporter quantitation (Fig. 1d), and then differential expression and comparative analyses (Fig. 1e).

We obtained a total of 31,865 unique peptides that mapped to 4259 unique mouse gene symbols. Of these, 4133 proteins were identified across the three groups (see Additional File 1: Table S1).. Not allowing any missing values, 3598 proteins were quantified. The distribution of protein abundance was highly skewed such that $5 \%$ of proteins $(n=211)$ accounted for $50 \%$ of the cumulative protein weight in the samples (Fig. 2a). Most of these highly abundant proteins were housekeeping and cytoskeletal proteins such as Atp1a3 ( $\alpha 3$ subunit of $\mathrm{Na}^{+}$/ $\mathrm{K}^{+}$ATPase), Sptan1 ( $\alpha$ spectrin), Actb ( $\beta$ actin), Sptbn1 ( $\beta$ spectrin) and Tubb4b (beta4 tubulin). Using a previously published purified mouse brain and cell type-specific proteome as a reference (7,198 identified and quantified proteins) [8], 3379 proteins identified in our dataset were also identified in the reference microglial proteome. Of these, 156 proteins were highly abundant ( $\geq 90$ th percentile of abundance) in both datasets. Overall, there was modest correlation of expression levels between the reference microglial proteome and our microglial proteome (Fig. 2b, Spearman's rank correlation $R=0.28$ for all proteins in common, Spearman's $R=0.23$ for the top 25th percentile in common). This modest correlation suggests possibility of methodological and age-related differences in microglial protein expression and myelination, since our proteome was derived from significantly older mice (26-30 weeks of age) as compared to the reference proteome (8-9 weeks of age). In the reference proteome, 633 microglia-specific proteins were also identified as having highest expression in microglia as compared to neurons or other glial cells. Of these, 189 proteins were identified in our microglial dataset. Among the most highly abundant proteins in both microglial proteomes $(\geq 80$ th percentile of abundance), 17 proteins also met criteria for microglia-specific expression (Fig. 2c). These proteins consistent across two independent datasets (including Myh9, Msn, Coro1a, Cotl1 and Bin1), represent the most highly abundant and microglial-specific proteins in mice.

We also performed gene ontology (GO) enrichment analyses on the entire list of 4133 proteins and identified 180 significant GO terms (adjusted $p$-value $<0.05$ ). The top 10 GO terms for each category are shown in Fig. 2d (also see Additional file 2: Table S2). This GO analysis shows that our microglial proteome was enriched for cytosolic/cytoplasmic proteins involved in transport and several catabolic, nucleotide/nucleoside 
a

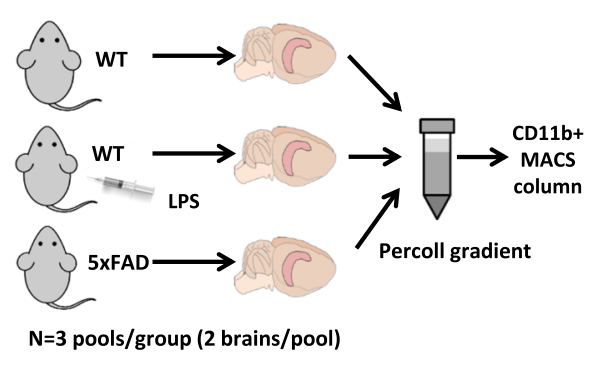

$\mathrm{N}=3$ pools/group (2 brains/pool) b

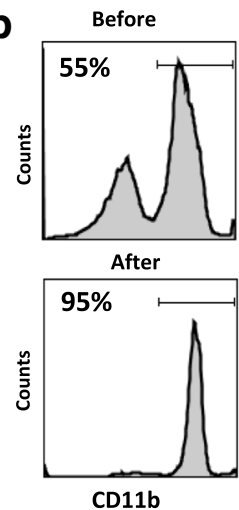

C

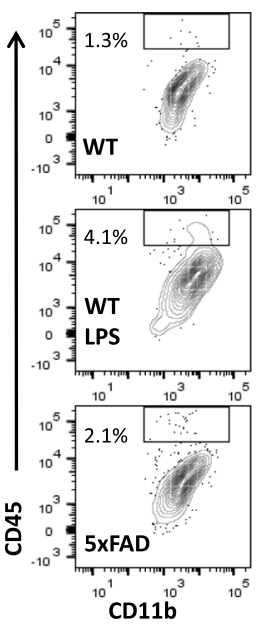

e

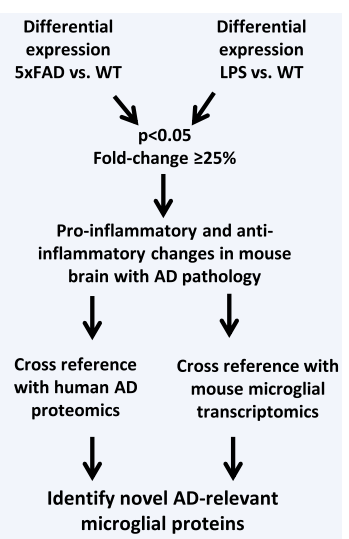

Fig. 1 Study design and analytic approach for comprehensive quantitative proteomic analysis of murine microglia. a Work-flow summarizing isolation and purification of acutely isolated CD11 b CNS immune cells from untreated 6-7 mo WT (C57BL/6 J) mice, LPS-treated WT mice (4 daily i.p. doses, $20 \mu \mathrm{g} / \mathrm{dose}$ ) and age/sex-matched 5xFAD transgenic mice were ( $N=3$ pools per group, 2 brains per pool). Following percoll density centrifugation, cells were enriched for $\mathrm{CD}_{11} \mathrm{~b}^{+}$cells by MACS magnetic separation. $\mathbf{b}$ Flow cytometric confirmation of successful enrichment of CD11 $\mathrm{b}^{+}$cells after MACS enrichment. Pre-enrichment (top), CD11 b+ cells account for 50-60\% of all live CNS mononuclear cells. Post-enrichment (bottom), CD11 b cells account for $>95 \%$ of all live cells. c Flow cytometric confirmation of minimal presence of peripherally derived CD11b + CD45high CNS-infiltrating macrophages or perivascular macrophages in untreated WT, LPS-treated WT and 5xFAD mice. $\mathbf{d}$ Proteomic work-flow for tandem mass tag (TMT) mass spectrometry based quantification. e Analytic approach used for differential expression analyses of datasets

metabolic and mitochondrial processes. Interestingly, "neuron projection" and "synapse" were also identified as significantly enriched GO terms in this dataset, which may be explained by the known role of microglia in neuronal synaptic pruning $[27,28]$. WikiPathways enriched in our microglial proteome included electron transport chain, oxidative phosphorylation/TCA cycle, proteasome degradation, glycolysis/gluconeogenesis, fatty acid beta oxidation and G protein signaling pathways (Fig. 2e, Additional file 2: Table S2). Results from GO analyses were not affected by the choice of background proteins used (all known mouse gene symbols or 11,937 proteins identified in a reference mouse brain proteome) [8]. In summary, the global microglial proteome from adult mice shows that the large majority of microglial proteins are ubiquitously expressed housekeeping proteins although highly abundant and microglia-specific proteins also exist. The observed enrichment of glycolytic, proteasome degradation, mitochondrial oxidative phosphorylation and fatty acid beta oxidation pathways suggest that adult microglia represent a highly metabolically active and dynamic group of glial cells, rather than bystander resting cells as they were once perceived to be.

\section{Differential expression and comparative analyses of} microglia in mouse models of acute neuroinflammation, $A D$ pathology and advanced aging

Of the 4133 proteins identified across all three groups, 187 proteins were differentially expressed in 5xFAD 


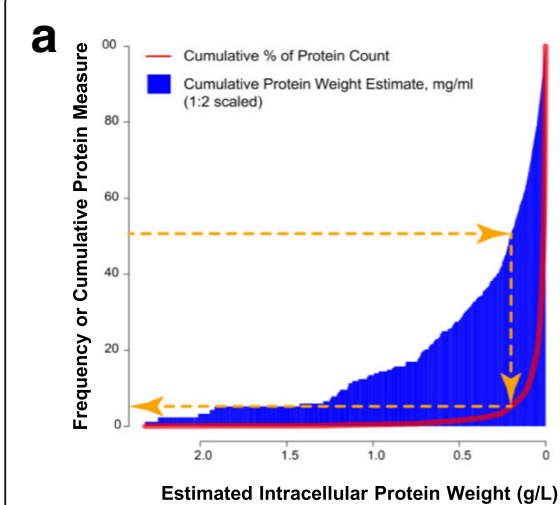

Estimated Intracellular Protein Weight (g/L)

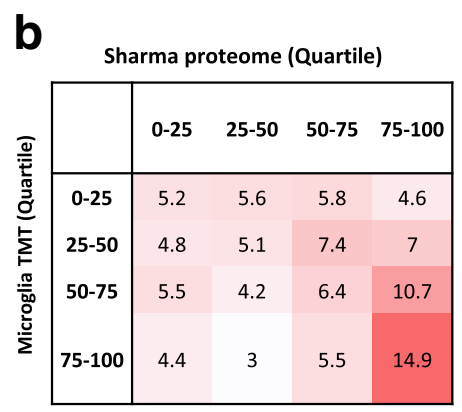

\section{d GO (Top 10 per category)} generation of precursor metabolites and energy cellular catabolic process

nucleobase, nucleoside and nucleotide metabolic process establishment of localization in cellular component organization heterocycle metabolic process translational elongation dicarboxylic acid metabolic process cytoskeletal protein binding GTPase activity $\mathrm{NADH}$ dehydrogenase activity NAD binding cation-transporting ATPase activity hydrogen ion transmembrane transporter activity GTP binding protein domain specific binding unfolded protein binding oxidoreductase activity cytoplasmic part organelle inner membrane

\section{Ontology type}

$\square$ Biological Process

$\square$ Molecular Function

- Cellular Component

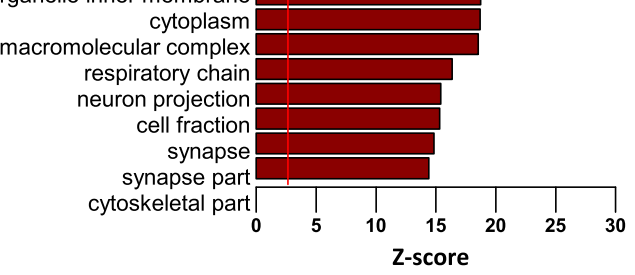

C

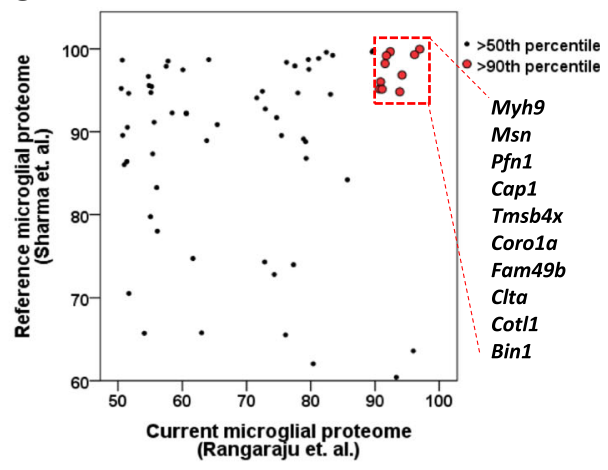

e

Wikipathways (Top 10)

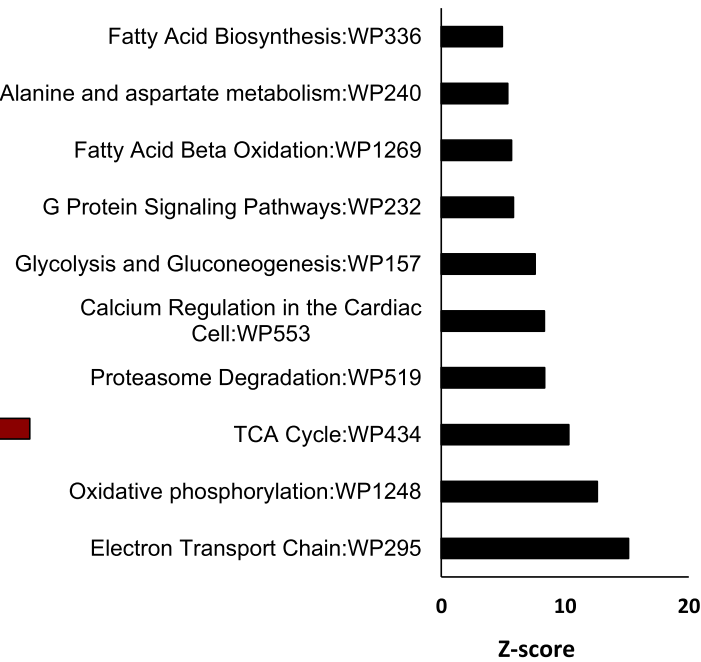

Fig. 2 A high-level summary, gene ontology and pathway analysis of 4133 microglial proteins identified by TMT. a Histogram representing the distribution of microglial proteins sorted by protein wet weight (bins with proteins contributing the most wet weight are on the left). Individual protein wet weight contributions were estimated using the Perseus Proteomic Ruler analysis plugin [66], which uses measurements of histone abundance to normalize reporter abundances assuming a total wet weight density of $200 \mathrm{mg} / \mathrm{ml}$. The cumulative distribution of protein abundance was highly dependent on a minority of proteins (of 4133 total) such that a majority of cumulative protein mass was accounted for by a small number of proteins, i.e., $5.1 \%$ of all proteins accounted for $50 \%$ of cumulative protein mass. b Comparison of relative abundance (abundance percentile rank) of proteins identified in our dataset with a reference mouse microglial proteome [8]. Abundance values of proteins identified in WT mouse microglia were binned into quartiles and compared with the reference proteome. The proportions (\%) of microglial proteins are shown in each box. c Scatter plot representation of the most abundant proteins identified in our microglial proteome and the reference microglial proteome. Proteins that were among the top 10th percentile of abundance in both datasets are highlighted in red. $\mathbf{d}$ Results from Gene Ontology (GO) enrichment analysis of 4133 identified microglial proteins against a background list of all known mouse gene symbols (or 11,937 proteins in a total brain reference proteome) [8]. The top $10 \mathrm{GO}$ terms from each of the three GO groups (Green: biological process, Purple: molecular function, Brown: cellular component) are shown. Degree of enrichment of each GO term is indicated by the Z-score (X-axis). Nearly identical results were obtained either background list of all mouse gene symbols or 11,937 mouse proteins identified in a total brain reference proteome. e Top 10 enriched functional pathways (WikiPathways) identified by enrichment analysis of 4133 microglial proteins

microglia and 1230 proteins were differentially expressed following LPS treatment in WT mice (Fig. 3, Additional file 3: Figure S1). Of 187 proteins differentially expressed in 5xFAD mice (Fig. 3a), 56 proteins demonstrated $\geq 1.25$-fold increased expression (top 5: App, Drg2,
Apoe, Nop56 and $\mathrm{Clu}$ ) while 105 proteins demonstrated $\geq 1.25$-fold decreased expression (top 5: Plekhg1, Lpgat1, Zc3h14, Ampd3 and Exd2). By applying Benjamini-Hochberg false-discovery-rate (FDR) correction to group-wise ANOVA $p$-values, 71 proteins 


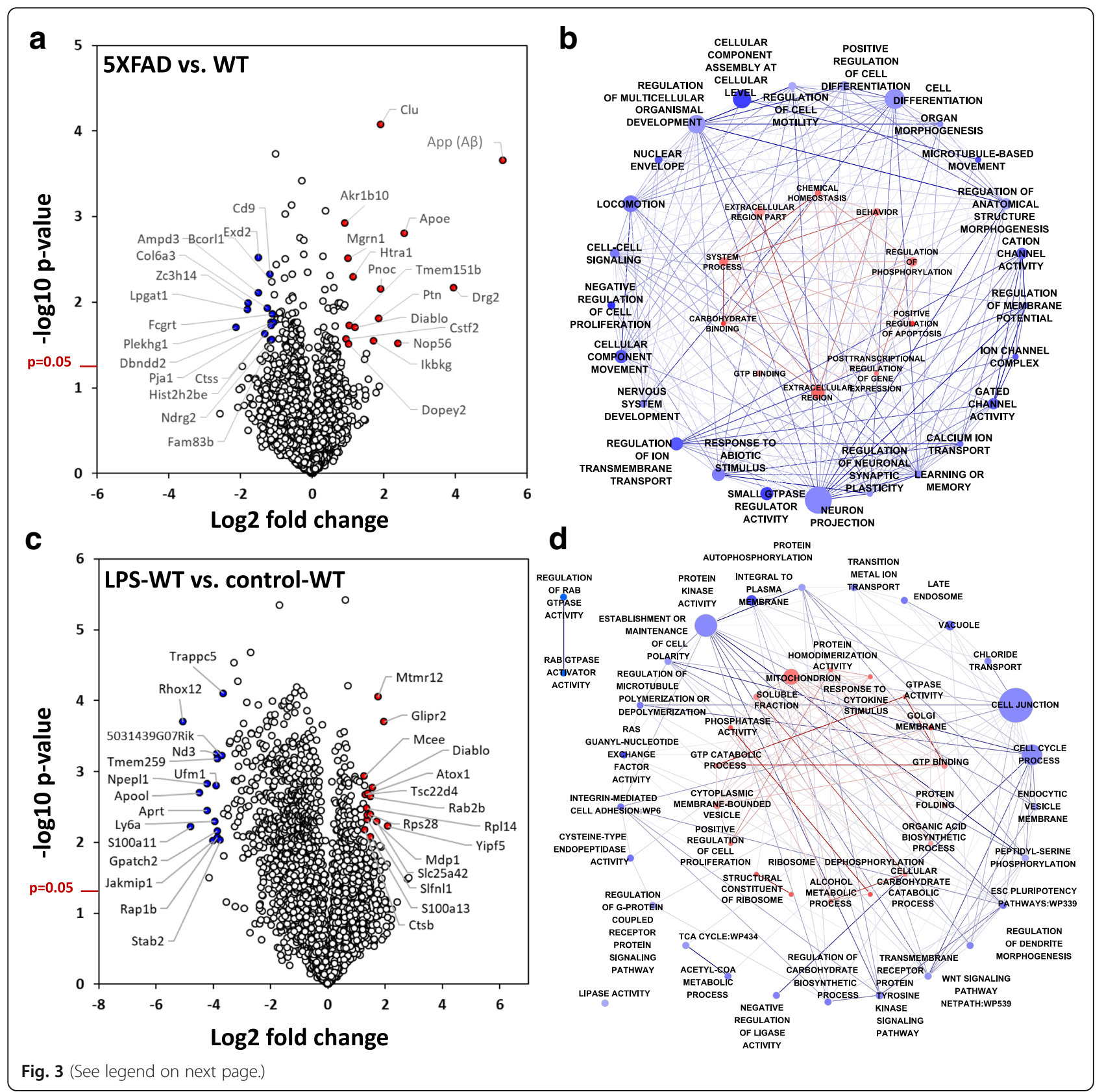




\begin{abstract}
(See figure on previous page.)
Fig. 3 Differential expression analyses of proteins identified in microglia derived from homeostatic, LPS-induced neuroinflammation and 5xFAD neurodegeneration mouse models. a Volcano plot representation of differentially expressed proteins comparing 5xFAD with untreated WT mouse microglia. The top 15 proteins with increased (red) and decreased (blue) expression are shown. X-axis represents $\log _{2}$ transformed fold change (5xFAD vs WT) and $y$-axis represents - $\log _{10}$ transformed $p$-value from a pairwise two-tailed T test comparing the two groups. $\mathbf{b}$ Gene enrichment map summarizing Gene Ontology (GO) terms and WikiPathways enriched in the 5xFAD vs WT differential expression analysis. Enrichment Maps were created in Cytoscape (Enrichment map plugin) Cytoscape using DAVID-formatted GO-Elite pruned Z-Score output tables. Red or Blue colors indicate significant GO terms (Z-score > 1.96) enriched in differentially expressed protein lists in 5xFAD microglia vs. WT microglia (Red: increased, Blue: decreased). Edge connectivity (Jaccard similarity metric) ranges from 0 (light color intensity) to 1 (dark red or blue intensity). Ontology network node colors represent the degree of average log2 fold change for all differentially expressed members of the ontology in the AD-WT networks. Since no overlap of gene symbols occurs between differential expression list-derived up and down sub-networks, respectively red and blue sub-networks were overlaid. Scale AD-CT: bluest $(-0.75)<->0$ (white) $<->$ reddest $\left(2.27 \log _{2}\right.$ Fold change). c Volcano plot representation of differentially expressed proteins comparing LPS-treated WT with untreated WT mouse microglia. The top 15 proteins with increased (red) and decreased (blue) expression are shown. X-axis represents $\log _{2}$ transformed fold change (LPS-treated WT vs untreated WT) and y-axis represents - $\log _{10}$ transformed p-value from a pairwise two-tailed T test comparing the two groups. $\mathbf{d}$ Gene enrichment map summarizing $\mathrm{GO}$ analysis of differentially expressed proteins in LPS-treated WT mouse microglia. Gene enrichments were created as described above using significant GO terms enriched in differentially expressed protein lists comparing microglia isolated from LPS-treated WT and untreated WT mice (Red: increased in LPS-treated WT mice; Blue: decreased in LPS-treated WT mice) microglial proteins. Scale LPS-WT/CT: bluest $(-2.21 \log 2$ FC) $<->0$ (white) $<->$ reddest (1.22 $\log _{2}$ fold change)
\end{abstract}

demonstrated significant differential expression (FDR < 0.05 ) across the three groups. Of these, 23 proteins were differentially expressed comparing $5 x F A D$ to WT microglia (6 up-regulated and 17 down-regulated) and the 6 proteins with increased expression in 5xFAD microglia included App, Apoe, Clu, Htra1, Rab2b and Necab1. GO analysis of differentially expressed proteins showed that extracellular proteins, carbohydrate binding, post-transcriptional regulation, GTP binding and regulation of phosphorylation were specifically enriched in proteins increased in 5xFAD microglia while extracellular proteins, anatomic structure development, ion channel activity, transmembrane transport inhibition of cell proliferation, locomotion and learning and memory were enriched GO terms in proteins decreased in 5xFAD microglia (Fig. 3b, Additional file 4: Table S3). Pathway analyses of differentially expressed proteins identified aberrant lipid trafficking, altered lipid metabolism and ATP metabolism as being enriched among proteins with increased expression in 5xFAD microglia while anti-inflammatory IL4 signaling was enriched in proteins with decreased expression in 5xFAD microglia (Additional file 5: Table S4). Transcriptional factors predicted to be upstream regulators of proteins overexpressed in 5xFAD microglia included PUR2 $\alpha$, AP-2 and p53 while Dec1 (Stra13) and c-Myc were predicted as upstream regulators of proteins decreased in 5xFAD microglia (Additional file 4: Table S3).

In addition to identifying neurodegeneration-associated microglial proteomic changes, we used the systemic LPS-induced neuroinflammation model to compare acute neuroinflammatory changes with chronic neuroinflammation seen in more indolent neurodegeneration models [12, 13]. In microglia from LPS-treated WT mice, 363 proteins were $\geq 1.25$-fold upregulated and 823 were $\geq 1.25$-fold down-regulated (Fig. 3c). Proteins increased following LPS treatment were enriched for GO terms including cellular responses to stress/cytokines, mitochondrial localization, increased cell proliferation and ribosomal processes (Fig. 3c, Additional file 4: Table S3). Conversely, proteins decreased by LPS treatment were enriched for GO terms including oxidoreductase activity, cell adhesion and cell contact, carboxylic acid metabolism and ATP metabolism, suggesting a general suppression of oxidative phosphorylation and motility (Fig. 3d, Additional file 2: Table S2). We identified several pro-inflammatory LPS-upregulated microglial proteins that were increased in $5 x F A D$ microglia (top 5: Clu, Nudt2, Glipr2, Diablo and Cstf2), and 30 LPS-downregulated proteins that were decreased in 5xFAD microglia (top 5: Bcorl1, Plekhg1, Rasgef1a, Ipo4 and Bicd1). Proteins differentially expressed in 5xFAD mouse microglia showed mostly concordant directions of change in microglia derived from LPS-treated WT mice (Fig. 4a, $R=0.47 p<0.001$ ) suggesting that $\mathrm{AD}$ pathology, in general, induces a pro-inflammatory state in microglia. However, differences between LPS-activated microglia and 5xFAD microglia were also observed. Several proteins were differentially expressed in only in 5xFAD mouse microglia without any LPS-induced changes, including increased levels of App (A $\beta$ peptide), Nop56, Ptn, Tmem151b and Mgrn1 and decreased levels of Hist2h2be, Sar1a, Ttc9a and Aimp3 (Fig. 4c), suggesting that these proteins may be uniquely relevant to $\mathrm{AD}$ pathology. A comparison of GO enrichment terms from each analysis (5xFAD vs WT and LPS-WT vs WT, Fig. 3) reveals that while both LPS and AD pathology resulted in a general inhibition of microglial motility/locomotion, LPS-treatment induced proliferative responses and mitochondrial metabolic processes while AD pathology inhibited cell differentiation and proliferation while promoting 


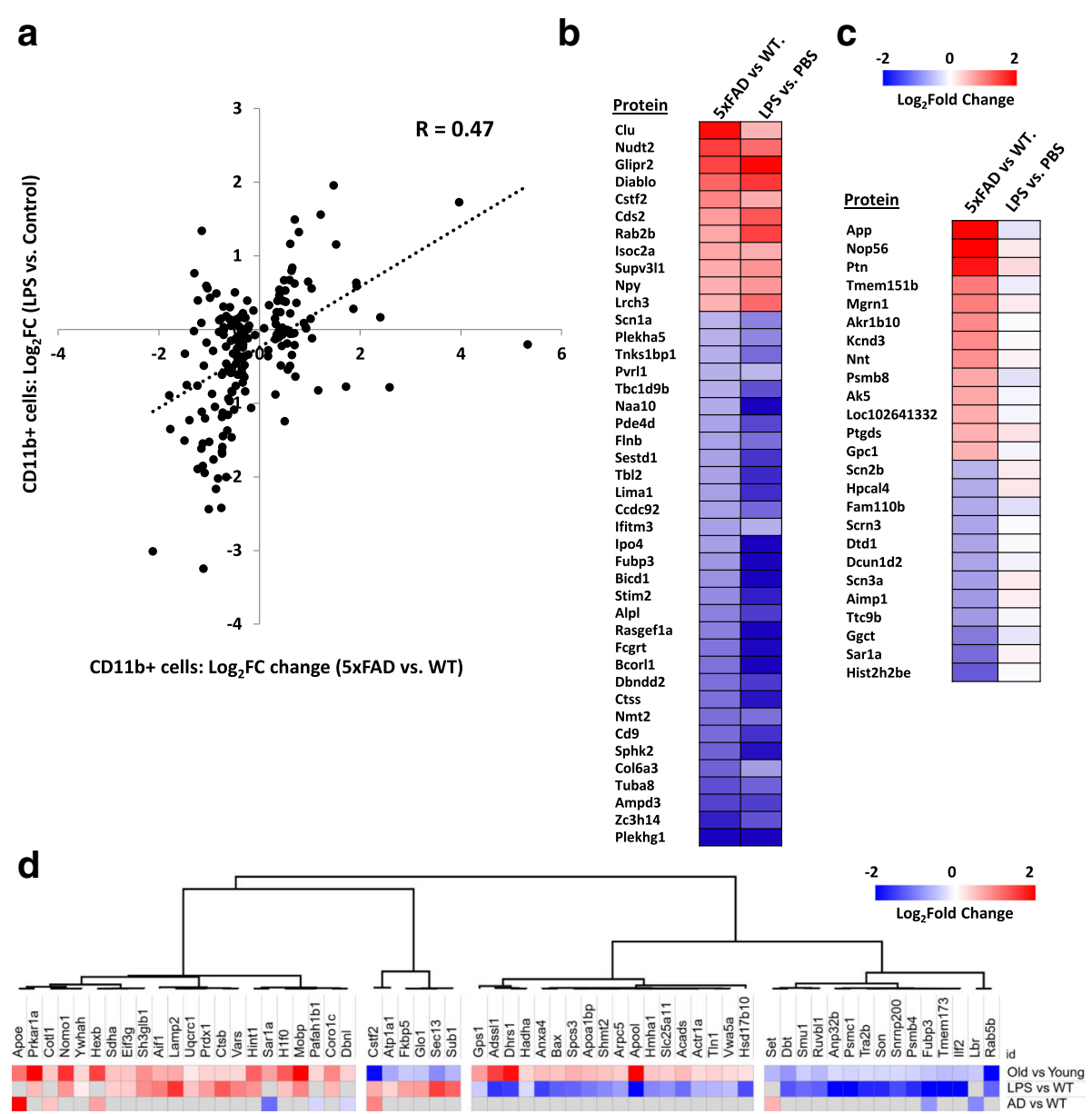

Fig. 4 Comparison of proteomic profiles of LPS-induced pro-inflammatory and 5xFAD AD pathological microglial states. a Scatter plot representation of relative expression (log2 transformed fold change) of all proteins differentially expressed in both LPS-treated WT (vs. untreated WT) as well as 5xFAD (vs WT) mouse models. Overall Pearson's correlation coefficient was $0.47, p<0.001$. $\mathbf{b}$ Heat map representing proteins with concordant changes in expression in microglia isolated from LPS-induced pro-inflammatory and 5xFAD neurodegeneration mouse models (compared to untreated WT microglia). c Heat map representing protein expression changes seen only in 5xFAD microglia but not in LPS-treated WT microglia. $\mathbf{d}$ Comparison of proteomic profiles of microglia from advanced aged mice with microglia derived from acute neuroinflammatory and 5xFAD neurodegeneration mouse models. Ageing-associated proteins and their relative expression data were obtained from an existing microglial proteome from young (age 3-4 mo) and aged (age 20-24 mo) WT C57BL/6 J mice [10]. Aging-associated proteins that were differentially expressed in either LPS-treated WT (vs WT) or 5xFAD (vs WT) conditions were included and an unsupervised hierarchical clustering analysis was performed to identify 4 distinct clusters. Grey boxes indicate proteins that were not significantly altered in the respective comparison

apoptosis, highlighting key metabolic and functional differences between these two states of microglial activation despite anatomical/structural similarities.

To compare the proteomic profile of microglia from acute neuroinflammation and neurodegeneration models with that of advanced aging, we contrasted our findings with an existing microglia proteomic study that compared young (3-5 mo) and very old mice (20-24 mo) [10]. Of 267 aging-associated microglial proteins identified in this reference dataset, 198 were identified in our microglial proteome of which 62 were differentially expressed either in 5xFAD or LPS-treated WT mouse microglia. These 62 proteins could be broadly classified into 4 clusters based on direction of change in protein expression in aged and LPS-treated mice (Fig. 4d). Only 8 proteins with altered expression in 5xFAD microglia also showed aging-related changes. Of the proteins increased in 5xFAD microglia, Hexb, Apoe and Cotl1 increased with aging while Cstf2 and Set decreased with aging. Interestingly, all three proteins that increased in aged WT and 5xFAD mouse microglia are highly expressed by microglia in the CNS [29], highlighting the microglial specificity of these $\mathrm{AD}$ - and aging-related protein alterations. In summary, this comparative microglial proteomic analysis from models of acute neuroinflammation, aging and $\mathrm{AD}$ pathology demonstrate model-specific as well as overlapping inflammatory changes in microglia. 


\section{Assessment of concordance between transcriptomic and proteomic changes in microglia in acute neuroinflammatory and neurodegenerative disease states} To assess the degree of concordance between transcriptomic and proteomic changes in microglia, we contrasted our proteomic findings in WT, LPS-induced neuroinflammation and 5xFAD models with existing RNAseq or

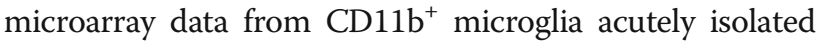
from similar model systems [5, 25]. Of the common gene symbols in existing RNAseq and our proteomic dataset from LPS-treated WT mouse microglia, we identified 72 genes/proteins were differentially expressed in both datasets of which only 35 (48.6\%) showed concordant changes (11 up-regulated, 24 down-regulated, Fig. 5a). Similarly, we contrasted our proteomics findings to existing tran-

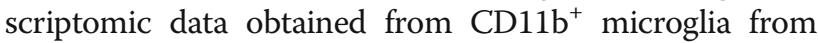
WT and 5xFAD mice [24], in which 3200 genes were found to be differentially expressed. Our proteomic dataset comparing 5xFAD to WT microglia included 902 of the 3200 genes which were altered at the transcript level. Among the 187 differentially expressed proteins (Fig. 5b), 43 were also altered at the transcriptomic level. Of these 43 , only $22(51.2 \%)$ showed concordant changes (8 up-regulated, 14 down-regulated). Among gene symbols identified in both proteomics and transcriptomics datasets, we also identified expression changes in 5xFAD mice that occurred exclusively at the protein or transcript levels (Fig. 5c). Proteins upregulated in 5xFAD microglia that did not demonstrate changes at the transcript level included Nop56, Pnoc, Ikbkg, Nudt2, Glipr2, Diablo and Htra1. Conversely, genes upregulated in 5xFAD microglia without proteome-level changes included Fabp3, Fabp5, Myo1e, Myo5a, Atp1a3, Lmnb1 and Clic4. Overall, these results highlight the modest levels of concordance between expression changes at the transcriptome and proteome levels and emphasize the need to perform molecular profiling at both transcript and protein levels since each can provide unique and novel biological insights into neurodegenerative disease processes.

\section{Identification of microglial proteomic changes in AD mouse models that are relevant to human AD}

Since amyloid $\beta$ accumulation seen in 5xFAD mice only represents one of the pathological hallmarks of human $\mathrm{AD}$, we do not expect all protein changes in mouse 5xFAD microglia to be relevant to human disease [26]. To identify proteomic changes observed in mouse $5 x F A D$ microglia that are consistent with human AD, we compared our mouse microglial proteome with an existing human post-mortem brain proteome in which frontal cortex from non-disease controls and pathology-confirmed AD cases were compared [14]. Three thousand six hundred forty-four proteins identified in our microglial proteome were also identified in this reference human brain proteome. While the overall correlation between relative protein expression data between mouse microglia and human brain samples for all identified proteins was low $\left(\mathrm{R}^{2}=0.002\right)$, moderate correlation $\left(R^{2}=0.34\right)$ was observed for 55 proteins that were differentially expressed in both datasets. Of these 55 proteins, 11 (including App, Apoe, Htra1, Cotl1 and $\mathrm{Clu}$ ) were concordantly increased while 23 (including Vgf, Rtn4, Alpl, Scn3a and Camk4) were concordantly decreased in both 5xFAD microglia and human $\mathrm{AD}$ brain (Fig. 6a).

Since App was identified as the most highly differentially expressed and human AD-relevant protein in 5xFAD microglia, we further investigated individual App peptide-level expression to determine whether this finding was driven by de-novo App synthesis or $A \beta$ phagocytosis by microglia. Five unique App peptides were identified in the dataset (Fig. 6b) of which 2 mapped to the $\mathrm{A} \beta$ sequence, 2 mapped to the $\mathrm{C}$-terminal YNPTY endocytosis motif and 1 mapped to $\mathrm{N}$-term residues 439-450. Since all five App peptides were identical in mice and humans, we were unable to directly confirm the source (mouse or human) of the App peptides identified in microglia. Selective increased expression of both $\mathrm{A} \beta 42$ peptides was noted in 5xFAD microglia while the $\mathrm{C}$-term peptide and the $\mathrm{N}$-term sequences showed no group-wise differences. This pattern of App peptide differential expression strongly suggests that intra-microglial $A \beta$ is phagocytosed and not produced within microglia.

\section{Validation of microglial Apoe and Cotl1 protein expression in $A D$}

Among the top 5 proteins (Fig. 6a) with increased expression in 5xFAD microglia and post-mortem human AD brain, Apoe and Cotl1 were also highly expressed at the transcriptional level by microglia $[8,29]$. Therefore, we performed additional neuropathological studies to characterize Cotl1 and Apoe protein expression in AD. Cotl1 or coactosin like F-actin binding protein 1 is an actin-binding protein that promotes the activity of 5-lipoxygenase, as enzyme that converts arachidonic acid into leukotriene A4 [30]. Cotl1 is also expressed by immune cells including macrophages and has been recently reported to regulate actin dynamics at the immune synapse in addition to promoting the function of lipoxygenase 5 (5LOX), an enzyme that converts arachidonic acid to leukotriene A4 (LTA4) [31]. Cotl1 is highly and specifically expressed by microglia at the transcript and protein levels (Fig. 7a) [8, 29], consistent with our finding that Cotl1 is a highly abundant microglial protein. In an analysis of an existing proteomic dataset comparing frontal cortical regions from AD, Parkinson's disease and non-disease post-mortem brain tissues [18], we found 


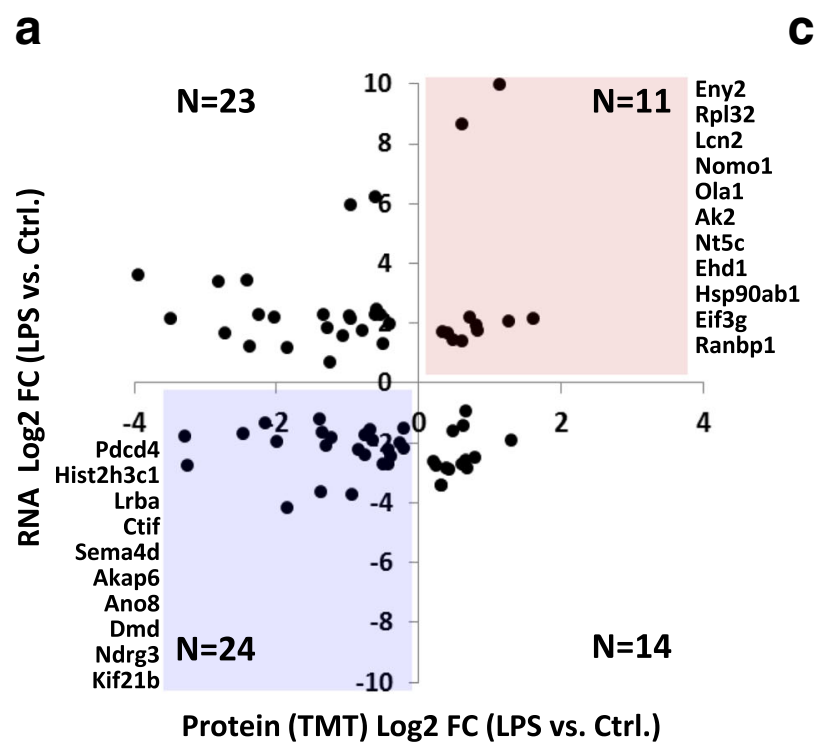

C 5 XFAD vs. WT CD11 $b^{+}$microglia
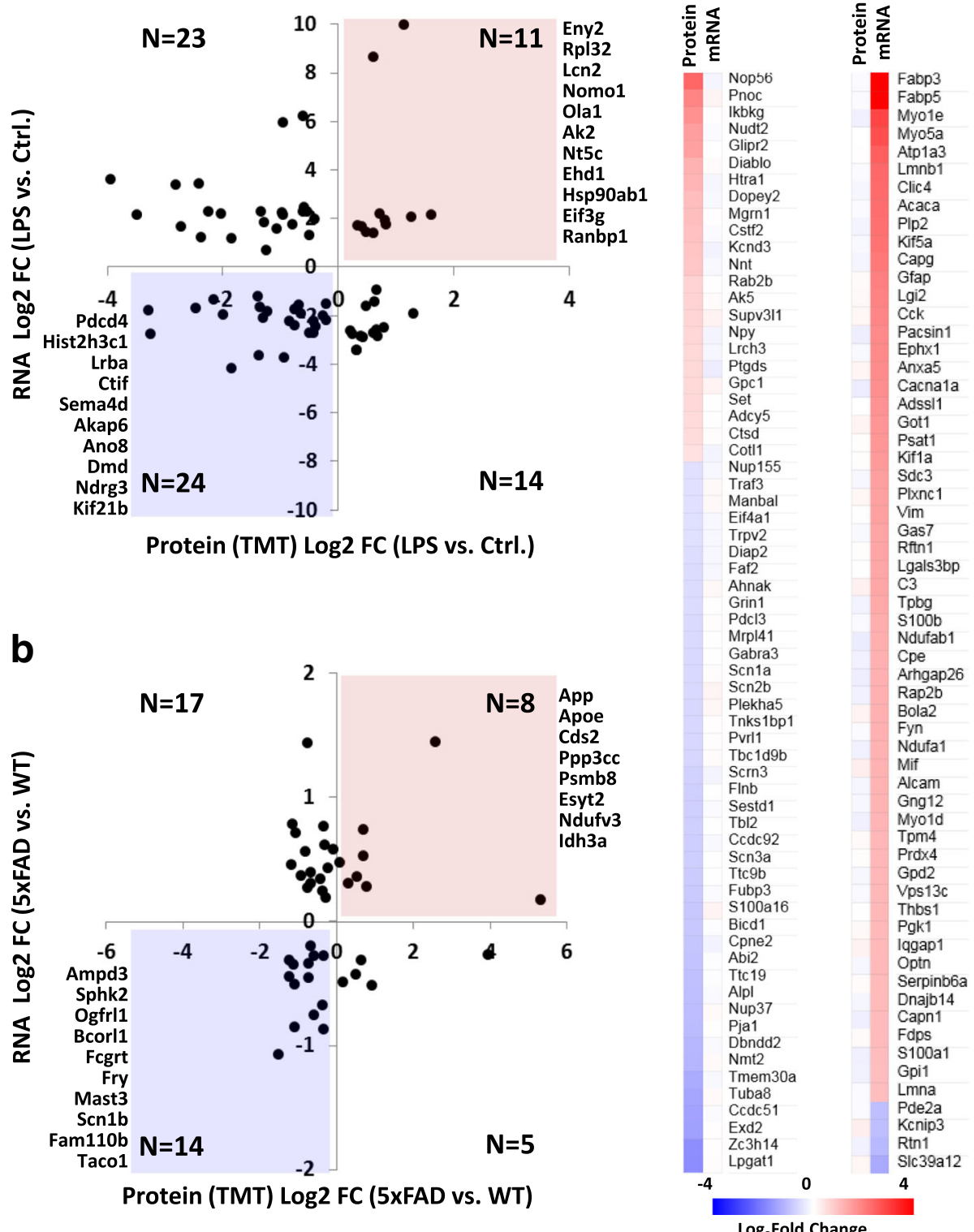

$\log _{2}$ Fold Change

Fig. 5 Modest concordance between transcriptomic and proteomic changes in microglia in acute neuroinflammatory and neurodegenerative disease conditions. a Scatter plot showing level of concordance between differentially expressed proteins and differentially expressed gene transcripts comparing LPS-treated WT to untreated WT microglia. The current microglial dataset was compared to transcriptomic data for shared gene symbols in a previously published RNA sequencing study of acutely isolated microglia from WT and LPS-treated WT mice [25]. Only differentially expressed proteins/transcripts in both datasets are shown. Gene symbols in the top right or bottom left quadrants indicate concordant changes. $\mathbf{b}$ Scatter plot showing level of concordance between differentially expressed proteins and differentially expressed gene transcripts comparing 5XFAD to WT microglia [24]. Our microglial proteomic dataset was cross referenced with an existing microarray gene expression study of acutely isolated WT and 5xFAD mouse microglia. Concordant changes are indicated in the top right and bottom left quadrants. c Heat map representing expression changes in 5xFAD microglia (vs. WT microglia) seen only at the protein level (left) or at the transcriptomic level (right)

that Cotl1 protein expression was increased in AD cases (Fig. 7b). Cotl1 protein expression was also strongly correlated with neurofibrillary tangle pathology (Braak stage) (Fig. 7c) and belonged to a glial co-expression protein module that was positively associated with $\mathrm{AD}$ pathology (Fig. 7d) [32, 33]. In addition to Cotl1, this glial module also contained other proteins that are pertinent to eicosanoid biosynthesis such as LTA4 hydrolase (Lta4H) which catalyzes the conversion of LTA4 to LTB4 [34] and, prostaglandin D2 synthase (Ptgds) which 

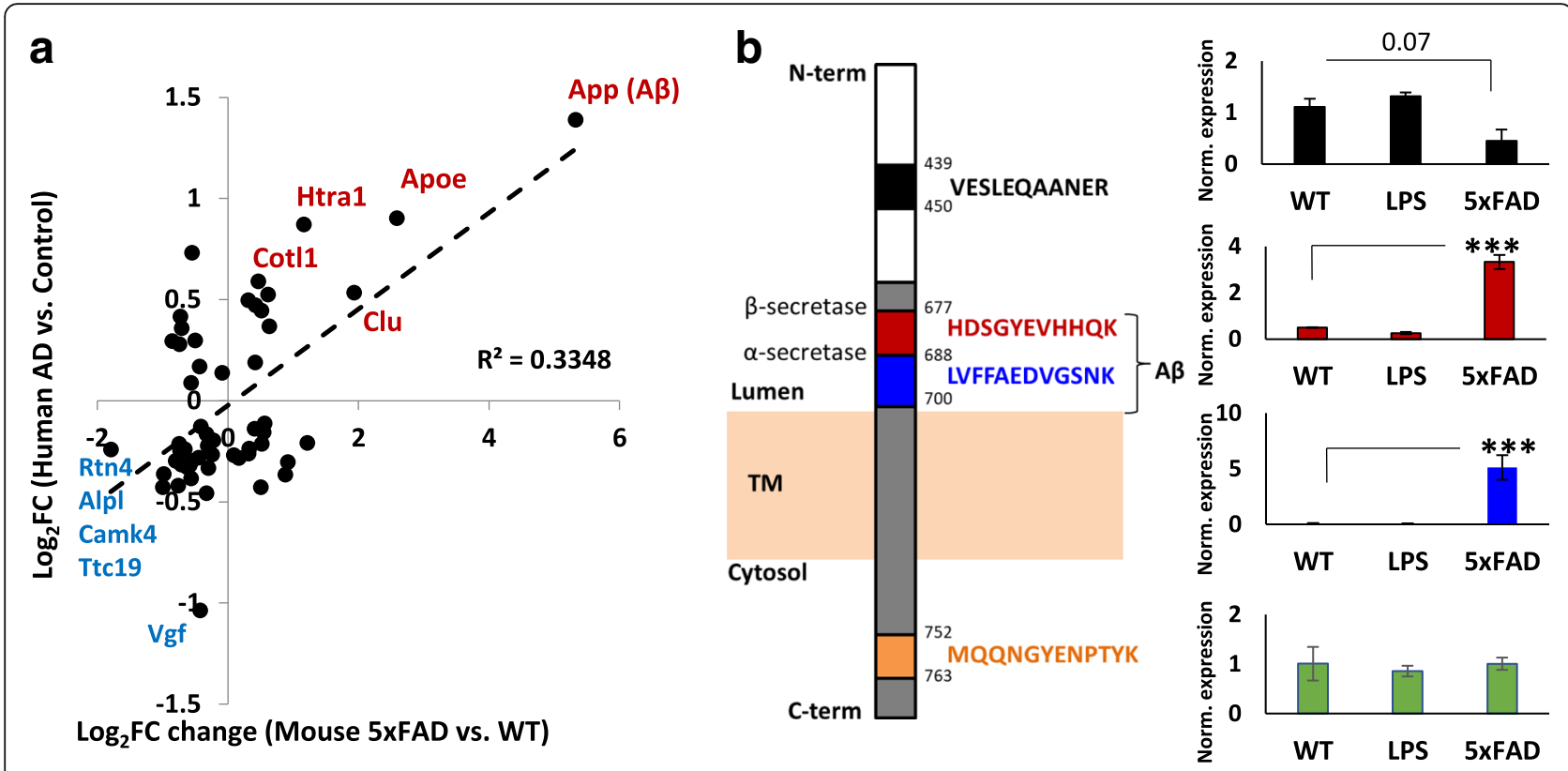

Fig. 6 Identification of microglial proteins with increased expression in 5xFAD mouse microglia with relevance to human AD pathology. a Scatter plot showing relative expression of proteins that were differentially expressed in both 5xFAD (vs WT) mouse microglia as well as a published human AD (vs non-AD non-disease control) whole brain proteomics dataset [18]. Pearson's correlation coefficient was determined to assess overall concordance. The top 5 concordantly increased (red) and decreased (blue) proteins are highlighted. Proteins shown in red indicate increased expression while blue proteins indicate decreased expression in both datasets. $\mathbf{b}$ Peptide-level analysis of all App peptides identified in the microglial proteome. Each unique peptide sequence is shown with a distinct color and each peptide's expression across all 3 mouse groups (relative to global internal standard) is shown. ${ }^{*} p<0.05,{ }^{* *} p<0.01$, ${ }^{* * *} p<0.005$

catalyzes the conversion of prostaglandin $\mathrm{H} 2$ (PG-H2) to PG-D2 [35]. In immuno-histochemical studies of human post-mortem brains, we found that Cotl1 was specifically expressed by $\mathrm{CD}^{+} 8^{+}$microglia (Fig. 7e) but not by $\mathrm{GFAP}^{+}$astrocytes (Fig. 7f). In a comparative immunohistochemistry analysis of AD and non-AD post-mortem frontal cortex tissues, we found that cortical and sub-cortical white matter microglia showed significantly higher Cotl1 protein expression as compared to non-AD controls (Fig. 7g). We also observed that microglia highly expressing Cotl1 in the AD brain had an activated morphology with larger cell bodies and numerous processes (Fig. 7g).

The second protein we validated was Apoe (apolipoprotein $E$ ) which regulates cholesterol and $A \beta$ transport into the brain and is strongly implicated in AD pathogenesis [36]. Carriers of the Apoc4 allele have significantly higher risk of developing late-onset $\mathrm{AD}$ and in mouse models of AD pathology, Apoe4 promotes neuroinflammatory responses leading to neurodegeneration, independent of its effects of $A \beta$ processing $[6,36,37]$. Apoe is highly expressed in astrocytes and microglia at both transcriptomic and proteomic levels (Fig. 8a) and is a specific marker of disease-associated-microglia that are highly prevalent in neurodegenerative diseases [5, 8, 29]. We observed that Apoe protein expression in mouse $5 x F A D$ brains closely resembled immunostaining of $A \beta$ plaques (Fig. 8b) which was not affected by preincubation with $A \beta$ fibrils (Additional file 3: Figure S2). Apoe also partly co-localized with Iba1 immuno-reactive microglia (Fig. 8b) confirming the presence of intra-microglial Apoe protein. Although Apoe and $A \beta$ (4G8) showed overlapping plaque-like immunostaining patterns with high degree of co-localization, Apoe within microglia also co-localized with $A \beta$, suggesting shared intracellular compartmentalization, presumably within phagosomes (Fig. 8c and d). These data suggest that Apoe within microglia could represent either phagocytic co-uptake of Apoe along with $\mathrm{A} \beta$, or may represent functional interaction between microglial Apoe in the phagocytic uptake of extra-cellular $A \beta$ and shared subsequent subcellular compartmentalization in microglia. These results show that Cotl1 is specific microglial protein that is selectively increased in AD while Apoe, at least at the protein level, may be involved in phagocytic uptake of $A \beta$ in plaque-associated microglia.

\section{Discussion}

Advances in deep RNA sequencing and proteomic methods have provided unprecedented opportunities to characterize the molecular and cellular complexity of the CNS [5, 8, 9, 25, 32, 38, 39]. Microglia are the principal immune effectors in the CNS [1] that perform homeostatic functions under normal conditions but adopt 


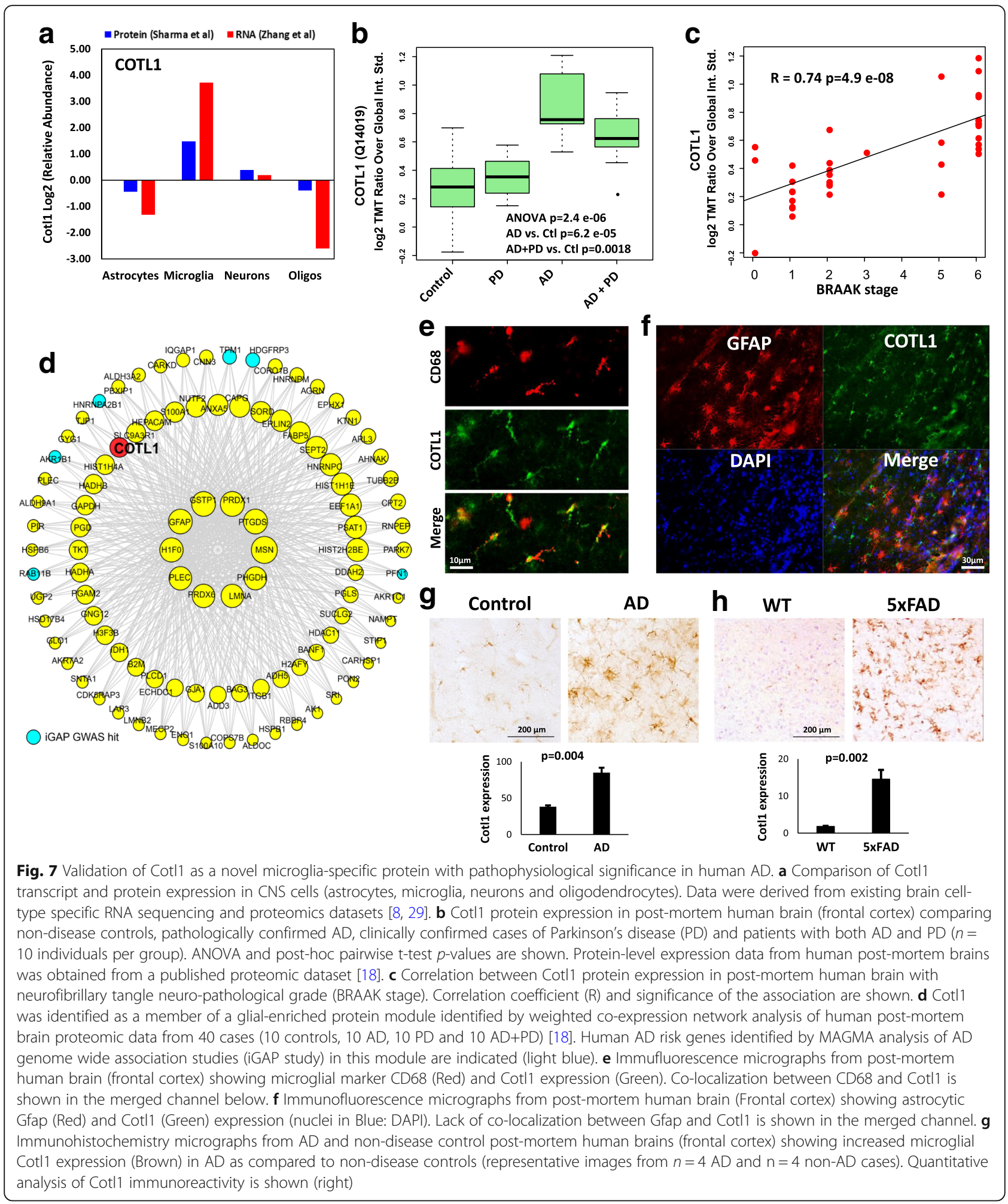

diverse activated profiles as revealed by recent whole-cell and single-cell transcriptomic studies $[5,6,8$, 25]. Several transcriptomic changes in microglia, however, may not reflect changes at the protein level due to post-transcriptional events that further regulate protein expression and degradation, leading to modest levels of agreement between transcript and protein expression data $[7,8,32]$. Primary microglial proteomes from 8 -week-old wild-type mice [8] and aged mice [10] as well as young adult $5 \mathrm{xFAD}$ mice (2-12 weeks of age) have 


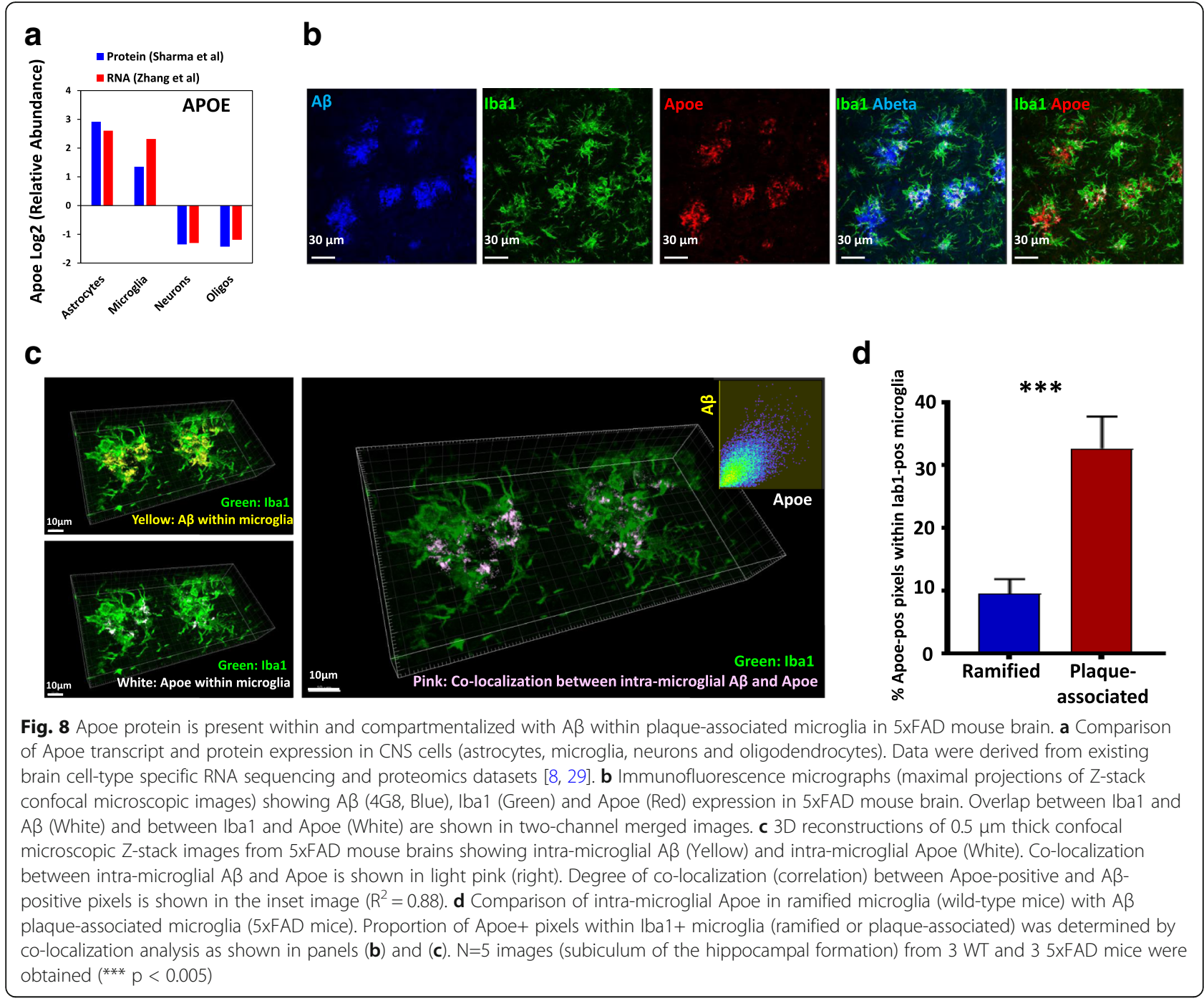

been published [11]. However, proteomic profiling of microglia from neuroinflammation models and later time points in neurodegenerative disease models has not been reported, representing a significant gap in the field. We present a comprehensive proteome of acutely isolated $\mathrm{CD}_{11 \mathrm{~b}^{+}}$microglia from older adult mice $(6-7 \mathrm{mo}$ og age) under normal, acute neuroinflammatory and chronic neurodegenerative disease conditions and highlight differentially expressed proteins specific to each state with relevance to human AD.

By contrasting microglia-specific changes in 5xFAD mice to existing human brain proteomes, we have found that App, Apoe, Clu, Htra1 and Cotll were among the most highly upregulated proteins in microglia isolated from a mouse model of AD pathology. Of these, App, Apoe, Clu and Htral have known AD-relevant biology although their expression and role within microglia in $\mathrm{AD}$ is not clear [36, 40,41]. Increased expression of Apoe, Htra1 and Clu proteins, but not of Cotl1, has been reported in 5xFAD microglia as early as 10 weeks of age when $A \beta$ deposition is first seen [11]. Additionally, we identified Cotl1 as a microglia-specific marker with increased expression in human AD. Cotl1 was also found in a recent network analysis study of post-mortem human $\mathrm{AD}$ brains to be a key member of a glial module that is positively correlated with AD pathology [32]. In this module, Cotl1 was co-expressed with leukotriene A4 hydrolase (Lt4ah). Since Cotl1 stabilizes and promotes the activity of lipoxygenase 5 (5-LOX) to convert arachidonic acid to LT-A4 [30,31], a co-upregulation of Lt4ah in AD microglia is predicted to facilitate LT-A4 conversion to LT-B4, a pro-inflammatory leukotriene implicated in neurotoxicity and $A \beta$ formation [42-44]. Another protein co-expressed with Cotl1 was PG-D2 synthase (Ptgds), an enzyme that catalyzes the conversion of PG-H2 to PG-D2 in the cyclooxygenase pathway of arachidonic acid metabolism [45]. Non-specific upstream inhibition of cyclooxygenase-2 (COX2) as well 
as specific inhibition of PG-D2 signaling results in neuroprotection in animal models [46, 47]. These converging lines of evidence suggest that at least some pro-inflammatory effects of microglia in $\mathrm{AD}$ may be mediated by LTB4 and PG-D2 [45]. Since Cotl1 is involved in actin dynamics, immune synapse function and regulation of eicosanoid biosynthesis, we suspect that Cotl1 may regulate motility and neuroinflammatory functions mediated by microglia in $\mathrm{AD}[30,31,45]$.

Apoe expression, like Cotl1, was also highly increased in 5xFAD microglia. This is consistent with observations from transcriptomic studies of microglia where Apoe was upregulated in neurodegenerative disease models and was identified as a marker of disease-associated-microglia [5, 6, 20]. Our immuno-histochemical studies show that Apoe protein, unlike Cotl1, is not expressed by ramified/ homeostatic microglia, but is seen within microglial processes that surround and infiltrate $A \beta$ plaques in $5 x F A D$ mice, and specifically co-localizes with intra-microglial $A \beta$. Since activated microglia engulf $A \beta$ and Apoe strongly co-localizes with $A \beta$ within microglia, our findings suggest that microglia may either phagocytose $A \beta$ and Apoe that co-aggregate in the $\mathrm{AD}$ brain or that Apoe's co-localization with $A \beta$ indicates functional interaction between $A \beta$ and Apoe during $A \beta$ phagocytosis and clearance $[48,49]$. In support of the latter possibility are recent findings demonstrating the role of Apoe, along with Trem2, in the transition of microglia from homeostatic to DAM states in $\operatorname{AD}[27,29]$, as well as the consistent identification of Apoe within microglia in our as well as other microglia proteomes $[8,10,11]$.

In addition to validating specific proteins with increased expression in 5xFAD microglia, our bioinformatics analyses provide clues regarding the general metabolic and inflammatory states of microglia in AD. We observed that dysfunctional lipid metabolism and trafficking seem to be features of microglia in AD brain. Since dysfunction in lipid metabolism and clearance are implicated in $\mathrm{AD}$ pathogenesis [50, 51], comprehensive lipidomic characterization of microglia from $A D$ models may provide novel insights into mechanisms of impaired lipid clearance in AD. Our observation of general suppression of IL4-related pathways in 5xFAD microglia along with similarities between protein expression profiles of microglia in 5xFAD mice and LPS-induced neuroinflammatory states suggest that microglia in $\mathrm{AD}$ adopt a profile more consistent with that of pro-inflammatory microglia. However, the identification of p53 and AP2 as upstream transcriptional regulators of $\mathrm{AD}$-associated microglial activation are distinct from regulators of canonical LPS-induced pro-inflammatory activation such [52-54] suggesting that unique inflammatory activation pathways are also upregulated by microglia in AD models. Beyond changes in AD models, our results from LPS-treated WT mice also demonstrate robust effects of systemic inflammation on energy metabolism and oxidative stress in microglia. GO analyses showed that LPS-induced systemic inflammation suppressed mitochondrial oxidative phosphorylation in microglia. In LPS-treated mouse microglia, 21 mitochondrial proteins were reduced while cellular stress response proteins were upregulated, suggesting that stress-induced mitochondrial damage may occur in microglia in response to systemic inflammation. Furthermore, LPS upregulated proteins involved in focal adhesion formation, proliferation, exocytosis and apoptosis confirming a state of microglial activation [13]. Since systemic LPS does not cross the blood brain barrier [55], our observations are more likely to represent indirect effects of LPS (involving Tnf or other soluble factors) rather than direct toll-like-receptor-mediated effects of LPS on microglia [56].

Our study also identified several highly abundant and microglia-specific proteins including Bin1, Msn, Cotl1 and Hexb [3]. The identification of Bin1 as a highly abundant protein in our microglial proteome agrees with a reference mouse microglial proteome [8] and is of particular relevance in $\mathrm{AD}$ [57]. At the transcript level, Bin1 is highly and specifically expressed by homeostatic microglia and Bin1 down-regulation occurs as microglia adopt DAM profiles in neurodegenerative disease [5, 25]. Although Bin 1 polymorphisms have been identified as independent risk factors for late-onset $\mathrm{AD}$ [57], the pathophysiological roles of Bin1 in $\mathrm{AD}$ are unclear. Based on our findings that are consistent with existing expression data for Bin 1 in the brain $[8,25]$, it may be particularly important to further investigate the role of Bin1 in microglia-mediated neuroinflammatory mechanisms in neurodegenerative disorders. We also identified Hexosaminidase B (HexB) as abundant microglial protein that was also previously shown to be microglia-specific at transcriptomic and proteomic levels $[3,5,8,25]$. We observed that HexB was upregulated by $5 x F A D$ microglia. Hexb is an endosomal-lsysomal enzyme that regulates ganglioside metabolism and is involved in autophagy [58]. Hexb mutation results in a rare lysosomal storage disorder called Type II GM2 gangliosidosis or Sandhoff disease that is characterized by progressive hearing loss, seizures, intellectual disabilities and motor deficits [59]. In the Hexb knock-out mouse, accumulation intra-neuronal $\mathrm{A} \beta$-like and synuclein-like protein aggregates [58]. Our findings of Hexb upregulation in microglia from $\mathrm{AD}$ mouse models further support the possibility that dysregulation of lipid metabolism, lysosomal functions and autophagy in microglia may contribute to $\mathrm{AD}$ neuropathology [60-62].

Despite the identification of microglia-specific proteins discussed above, some of the more well-known microglial 
proteins such as Tmem119 and P2ry12 were not identified in our study $[63,64]$. It is also interesting to note that the reference microglial proteome [8] did not identify Tmem119 in microglia even though Tmem119 protein expression by microglial has been confirmed by immuno-histochemical methods [25, 64]. This may be explained by our use of whole cell lysates, rather than membrane-enriched fractions for our study, leading to a lower likelihood of identifying cell membrane anchored proteins. This bias in our dataset is further supported by the lack of enrichment of GO terms such as "cell membrane" in our analyses. Another possibility for the relative absence of membrane proteins in our study is potential loss of cell membrane fragments during the cell isolation process, a likely result of heavy myelination and architectural complexity of the aging mouse brain as contrasted with post-natal or younger adult mice. Since membrane receptors, ion channels and transporters may represent drug targets for neurodegeneration, future studies using more advanced proteomics technologies with deeper coverage coupled with enrichment of membrane-associated microglial proteins will likely help overcome this limitation.

Additional limitations of this study also deserve discussion. In addition to ubiquitous and microglia-specific proteins, several proteins highly expressed by astrocytes, oligodendrocytes and neurons were also identified in our microglial proteome despite $>95 \%$ enrichment of CD11b ${ }^{+}$ cells in our samples. While this could indicate cell or protein contamination by adherent non-microglial cell fragments during $\mathrm{CD} 11 \mathrm{~b}^{+}$enrichment, this could also be indicative of constant pruning of neuronal synapses or dendrites as well as phagocytic uptake of cellular debris, myelin and astrocytic processes by microglia. Indeed, an age dependent increase in myelin basic protein (Mbp) peptides in mouse microglia has been previously reported [65], which is more likely to explain why Mbp was highly abundant in our dataset (top 10 percentile). As compared to the reference proteome in which over 7000 proteins were identified across 4 cell types by label-free quantitative methods [8], we identified 4133 proteins by TMT. This lower number of proteins identified could be attributed to lower microglial yield from the older mouse brain as compared to younger mice. Thus, pooling additional brain samples as well as enzymatic digestion prior to microglial isolation may be considered in future studies to improve protein yield. A more likely explanation for lower number of proteins identified is that our single batch of fractionated TMT, 10-plex-quantified by SPS-MS3 mass spectrometry did not contain non-microglial enriched samples from which to borrow or trigger identification and quantification of shared proteins which may be higher expressed in other cell types (i.e., neurons, oligodendrocytes and astrocytes). Lastly, our analyses using 3 pools per group were statistically underpowered to detect statistically significant changes using FDR corrections for pair-wise comparisons because of which we considered unadjusted $p$-values $<0.05$ as statistically significant. To overcome this inherent limitation, we applied an additional fold-change threshold of $25 \%$ which exceeded the overall coefficient of variation for all protein measurements (6-7\%) in each of the three groups.

\section{Conclusions}

In conclusion, this deep and comprehensive proteomic study of purified adult mouse microglia has allowed us to identify shared and unique microglial proteomic changes in acute neuroinflammatory, aging and $\mathrm{AD}$ mouse models in addition to identifying novel roles for microglial proteins in human neurodegeneration. In addition to serving as a valuable resource to the neuroscience research community, our study emphasizes the value of applying state-of-the-art proteomics methods as a complimentary approach to transcriptomics, to provide novel molecular insights in neurodegeneration.

\section{Additional files}

Additional file 1: Table S1. Log2 transformed protein abundance data
and differential expression analyses. (XLSX $1522 \mathrm{~kb}$ )
Additional file 2: Table S2. GO Elite analysis of all 4,133 proteins
identified in mouse microglia by TMT mass spectrometry. (XLSX $63 \mathrm{~kb}$ )
Additional file 3: Supplemental Figures. Figure S1. Hierarchical
clustering of proteins differentially expressed ( $p<0.05)$ in either WT-LPS vs
WT-control and 5xFAD vs WT comparisons. Figure S2. Pre-incubation of
anti-Apoe antibody with fibrillar Aß does not abolish plaque-like immuno-
staining for Apoe. (DOCX $761 \mathrm{~kb})$

Additional file 4: Table S3. GO Elite analysis of differentially expressed proteins in WT, LPS-treated WT and 5xFAD mouse microglia. (XLSX 20 kb)

Additional file 5: Table S4. Pathway analysis of proteins differentially increased in 5xFAD (vs. WT) microglia. (XLSX $18 \mathrm{~kb}$ )

\section{Abbreviations}

AD: Alzheimer's disease; Aß: Amyloid beta; DAM: Disease-associatedmicroglia; LPS: Lipopolysaccharide

\section{Funding}

Work supported by Emory Alzheimer's Disease Research Center Grant P50 AG025688, American Brain Foundation (SR \#28301), Alzheimer's Association (SR \#37102), NINDS (SR: K08-NS099474-1; CMH: K08-NS087121) and Emory Neuroscience NINDS Core facilities (P30 NS055077). Other NIH grants that supported this work include U01AG046161, RF1AG057471, R01AG057330 and RF1AG057470, 5R01AG053960. NTS was also supported by Alzheimer's Association grant 11060 .

\section{Availability of data and materials}

The mass spectrometry proteomics data have been deposited to the ProteomeXchange Consortium via the PRIDE partner repository with the dataset identifier PXD009137. Human brain proteomic data that has been previously published is publicly available $[14,18]$.

\section{Authors' contributions}

Conceptualization: SR, EBD, SAR, NTS and AIL; Methodology: SR, EBD, SAR, NTS, AlL; Investigation: SR, EBD, SAR, DD, CMH, TG, JW, HX, PR and HX; Writing-Original draft: SR, EBD; Writing-Review and Editing: SR, EBD, SAR, CMH, AlL, JJ, RB, NTS; Funding Acquisition: SR, AlL, NTS; Resources: AlL, NTS, JJL; Supervision: AlL, NTS. 


\section{Ethics approval and consent to participate}

Approval from the Emory University Institutional Animal Care and Use Committee was obtained prior to all animal-related studies (IACUC protocol \# 300123).

\section{Consent for publication}

All authors have approved of the contents of this manuscript and provided consent for publication.

\section{Competing interests}

The authors declare that they have no competing interests.

\section{Publisher's Note}

Springer Nature remains neutral with regard to jurisdictional claims in published maps and institutional affiliations.

\section{Author details}

'Department of Neurology, Emory University, Atlanta, GA 30322, USA.

${ }^{2}$ Department of Biochemistry, Emory University, Atlanta, GA 30322, USA.

\section{Received: 13 March 2018 Accepted: 18 June 2018}

\section{Published online: 28 June 2018}

\section{References}

1. Ginhoux F, Lim S, Hoeffel G, Low D, Huber T. Origin and differentiation of microglia. Front Cell Neurosci. 2013:7:45.

2. Li Q, Barres BA. Microglia and macrophages in brain homeostasis and disease. Nat Rev Immunol. 2018;18(4):225-242.

3. Crotti A, Ransohoff RM. Microglial physiology and pathophysiology: insights from genome-wide transcriptional profiling. Immunity. 2016:44:505-15.

4. Prinz M, Priller J. Microglia and brain macrophages in the molecular age: from origin to neuropsychiatric disease. Nat Rev Neurosci. 2014;15:300-12.

5. Keren-Shaul H, Spinrad A, Weiner A, Matcovitch-Natan O, Dvir-Szternfeld R, Ulland TK, David E, Baruch K, Lara-Astaiso D, Toth B, et al. A unique microglia type associated with restricting development of Alzheimer's disease. Cell. 2017;169:1276-90. e1217

6. Krasemann S, Madore C, Cialic R, Baufeld C, Calcagno N, El Fatimy R, Beckers L, O'Loughlin E, Xu Y, Fanek Z, et al. The TREM2-APOE pathway drives the transcriptional phenotype of dysfunctional microglia in neurodegenerative diseases. Immunity. 2017;47:566-81. e569

7. Lundberg E, Fagerberg L, Klevebring D, Matic I, Geiger T, Cox J, Algenas C, Lundeberg J, Mann M, Uhlen M. Defining the transcriptome and proteome in three functionally different human cell lines. Mol Syst Biol. 2010;6:450.

8. Sharma K, Schmitt S, Bergner CG, Tyanova S, Kannaiyan N, Manrique-Hoyos N, Kongi K, Cantuti L, Hanisch UK, Philips MA, et al. Cell type- and brain region-resolved mouse brain proteome. Nat Neurosci. 2015;18:1819-31.

9. Umoh ME, Dammer EB, Dai J, Duong DM, Lah JJ, Levey Al, Gearing M, Glass JD, Seyfried NT. A proteomic network approach across the ALS-FTD disease spectrum resolves clinical phenotypes and genetic vulnerability in human brain. EMBO Mol Med. 2018;10:48-62.

10. Flowers A, Bell-Temin H, Jalloh A, Stevens SM Jr, Bickford PC. Proteomic anaysis of aged microglia: shifts in transcription, bioenergetics, and nutrient response. J Neuroinflammation. 2017;14:96.

11. Boza-Serrano A, Yang Y, Paulus A, Deierborg T. Innate immune alterations are elicited in microglial cells before plaque deposition in the Alzheimer's disease mouse model 5xFAD. Sci Rep. 2018:8:1550

12. Chen Z, Jalabi W, Shpargel KB, Farabaugh KT, Dutta R, Yin X, Kidd GJ, Bergmann CC, Stohlman SA, Trapp BD. Lipopolysaccharide-induced microglial activation and neuroprotection against experimental brain injury is independent of hematogenous TLR4. J Neurosci. 2012;32:11706-15.

13. Rangaraju S, Raza SA, Pennati A, Deng Q, Dammer EB, Duong D, Pennington MW, Tansey MG, Lah JJ, Betarbet R, et al. A systems pharmacology-based approach to identify novel Kv1.3 channel-dependent mechanisms in microglial activation. J Neuroinflammation. 2017;14:128.

14. Ping L, Duong DM, Yin L, Gearing M, Lah JJ, Levey Al, Seyfried NT. Global quantitative analysis of the human brain proteome in Alzheimer's and Parkinson's Disease. Sci Data. 2018;5:180036

15. Wingo TS, Duong DM, Zhou M, Dammer EB, Wu H, Cutler DJ, Lah JJ, Levey Al, Seyfried NT. Integrating next-generation genomic sequencing and mass spectrometry to estimate allele-specific protein abundance in human brain. J Proteome Res. 2017;16:3336-47.
16. Kall L, Canterbury JD, Weston J, Noble WS, MacCoss MJ. Semi-supervised learning for peptide identification from shotgun proteomics datasets. Nat Meth. 2007;4:923-5.

17. Zambon AC, Gaj S, Ho I, Hanspers K, Vranizan K, Evelo CT, Conklin BR, Pico AR, Salomonis N. GO-elite: a flexible solution for pathway and ontology over-representation. Bioinformatics. 2012;28:2209-10.

18. Seyfried NT, Dammer EB, Swarup V, Nandakumar D, Duong DM, Yin L, Deng Q, Nguyen T, Hales CM, Wingo T, et al. A multi-network approach identifies protein-specific co-expression in asymptomatic and symptomatic Alzheimer's disease. Cell Syst. 2017;4:60-72. e64

19. Merico D, Isserlin R, Stueker O, Emili A, Bader GD. Enrichment map: a network-based method for gene-set enrichment visualization and interpretation. PLoS One. 2010;5:e13984.

20. Rangaraju S, Dammer EB, Raza SA, Rathakrishnan P, Xiao H, Gao T, Duong DM, Pennington MW, Lah JJ, Seyfried NT, Levey Al. Identification and therapeutic modulation of a pro-inflammatory subset of disease-associatedmicroglia in Alzheimer's disease. Mol Neurodegener. 2018;13(1):24

21. Casey CS, Atagi Y, Yamazaki Y, Shinohara M, Tachibana M, Fu Y, Bu G, Kanekiyo T. Apolipoprotein E inhibits cerebrovascular Pericyte mobility through a RhoA protein-mediated pathway. J Biol Chem. 2015;290:14208-17.

22. Thul PJ, Akesson L, Wiking M, Mahdessian D, Geladaki A, Ait Blal H, Alm T, Asplund A, Bjork L, Breckels LM, et al. A subcellular map of the human proteome. Science. 2017;26;356(6340). https://doi.org/10.1126/science.aal3321.

23. Rangaraju S, Gearing M, Jin LW, Levey A. Potassium channel Kv1.3 is highly expressed by microglia in human Alzheimer's disease. J Alzheimers Dis. 2015:44:797-808.

24. Wang Y, Cella M, Mallinson K, Ulrich JD, Young KL, Robinette ML, Gilfillan S, Krishnan GM, Sudhakar S, Zinselmeyer BH, et al. TREM2 lipid sensing sustains the microglial response in an Alzheimer's disease model. Cell. 2015; 160:1061-71.

25. Bennett ML, Bennett FC, Liddelow SA, Ajami B, Zamanian JL, Fernhoff NB, Mulinyawe SB, Bohlen CJ, Adil A, Tucker A, et al. New tools for studying microglia in the mouse and human CNS. Proc Natl Acad Sci U S A. 2016; 113:E1738-46.

26. Oakley H, Cole SL, Logan S, Maus E, Shao P, Craft J, Guillozet-Bongaarts A, Ohno M, Disterhoft J, Van Eldik L, et al. Intraneuronal beta-amyloid aggregates, neurodegeneration, and neuron loss in transgenic mice with five familial Alzheimer's disease mutations: potential factors in amyloid plaque formation. J Neurosci. 2006;26:10129-40.

27. Paolicelli RC, Bolasco G, Pagani F, Maggi L, Scianni M, Panzanelli P, Giustetto $M$, Ferreira TA, Guiducci E, Dumas $L$, et al. Synaptic pruning by microglia is necessary for normal brain development. Science. 2011;333:1456-8.

28. Hong S, Dissing-Olesen L, Stevens B. New insights on the role of microglia in synaptic pruning in health and disease. Curr Opin Neurobiol. 2016;36:128-34.

29. Zhang Y, Chen K, Sloan SA, Bennett ML, Scholze AR, O'Keeffe S, Phatnani HP, Guarnieri P, Caneda C, Ruderisch N, et al. An RNA-sequencing transcriptome and splicing database of glia, neurons, and vascular cells of the cerebral cortex. J Neurosci. 2014:34:11929-47.

30. Esser J, Rakonjac M, Hofmann B, Fischer L, Provost P, Schneider G, Steinhilber D, Samuelsson B, Radmark O. Coactosin-like protein functions as a stabilizing chaperone for 5-lipoxygenase: role of tryptophan 102. Biochem J. 2009:425:265-74.

31. Kim J, Shapiro MJ, Bamidele AO, Gurel P, Thapa P, Higgs HN, Hedin KE, Shapiro VS, Billadeau DD. Coactosin-like 1 antagonizes cofilin to promote lamellipodial protrusion at the immune synapse. PLoS One. 2014;9:e85090.

32. Xu P, Duong DM, Seyfried NT, Cheng D, Xie Y, Robert J, Rush J, Hochstrasser M, Finley D, Peng J. Quantitative proteomics reveals the function of unconventional ubiquitin chains in proteasomal degradation. Cell. 2009;137:133-45.

33. Braak H, Alafuzoff I, Arzberger T, Kretzschmar H, Del Tredici K. Staging of Alzheimer disease-associated neurofibrillary pathology using paraffin sections and immunocytochemistry. Acta Neuropathol. 2006;112:389-404.

34. Lawrence T, Willoughby DA, Gilroy DW. Anti-inflammatory lipid mediators and insights into the resolution of inflammation. Nat Rev Immunol. 2002;2:787-95.

35. Taniguchi H, Mohri I, Okabe-Arahori H, Aritake K, Wada K, Kanekiyo T, Narumiya S, Nakayama M, Ozono K, Urade Y, Taniike M. Prostaglandin D2 protects neonatal mouse brain from hypoxic ischemic injury. J Neurosci. 2007;27:4303-12.

36. Liu CC, Liu CC, Kanekiyo T, Xu H, Bu G. Apolipoprotein E and Alzheimer disease: risk, mechanisms and therapy. Nat Rev Neurol. 2013;9:106-18. 
37. Guo L, LaDu MJ, Van Eldik LJ. A dual role for apolipoprotein e in neuroinflammation: anti- and pro-inflammatory activity. J Mol Neurosci. 2004;23:205-12.

38. Lowe R, Shirley N, Bleackley M, Dolan S, Shafee T. Transcriptomics technologies. PLoS Comput Biol. 2017;13:e1005457.

39. Thongboonkerd V, LaBaer J, Domont GB. Recent advances of proteomics applied to human diseases. J Proteome Res. 2014;13:4493-6.

40. Grau S, Baldi A, Bussani R, Tian X, Stefanescu R, Przybylski M, Richards P, Jones SA, Shridhar V, Clausen T, Ehrmann M. Implications of the serine protease HtrA1 in amyloid precursor protein processing. Proc Natl Acad Sci U S A. 2005;102:6021-6.

41. O'Brien RJ, Wong PC. Amyloid precursor protein processing and Alzheimer's disease. Annu Rev Neurosci. 2011;34:185-204.

42. Chu J, Pratico D. The 5-lipoxygenase as modulator of Alzheimer's gammasecretase and therapeutic target. Brain Res Bull. 2016;126:207-12.

43. Joshi YB, Pratico D. The 5-lipoxygenase pathway: oxidative and inflammatory contributions to the Alzheimer's disease phenotype. Front Cell Neurosci. 2014;8:436

44. Joshi YB, Di Meco A, Pratico D. Modulation of amyloid-beta production by leukotriene B4 via the gamma-secretase pathway. J Alzheimers Dis. 2014;38:503-6.

45. Manev H, Chen H, Dzitoyeva S, Manev R. Cyclooxygenases and 5lipoxygenase in Alzheimer's disease. Prog Neuro-Psychopharmacol Biol Psychiatry. 2011;35:315-9.

46. Iwasa K, Yamamoto S, Yagishita S, Maruyama K, Yoshikawa K. Excitotoxicityinduced prostaglandin D2 production induces sustained microglial activation and delayed neuronal death. J Lipid Res. 2017;58:649-55.

47. Mohri I, Taniike M, Taniguchi H, Kanekiyo T, Aritake K, Inui T, Fukumoto N, Eguchi N, Kushi A, Sasai H, et al. Prostaglandin D2-mediated microglia/ astrocyte interaction enhances astrogliosis and demyelination in twitcher. J Neurosci. 2006:26:4383-93.

48. Kanekiyo T, Xu H, Bu G. ApoE and Abeta in Alzheimer's disease: accidental encounters or partners? Neuron. 2014;81:740-54

49. Ries M, Sastre M. Mechanisms of Abeta clearance and degradation by glial cells. Front Aging Neurosci. 2016;8:160.

50. Visan I. Alzheimer's disease microglia. Nat Immunol. 2017:18:876.

51. Di Paolo G, Kim TW. Linking lipids to Alzheimer's disease: cholesterol and beyond. Nat Rev Neurosci. 2011;12:284-96.

52. Aloi MS, Su W, Garden GA. The p53 transcriptional network influences microglia behavior and Neuroinflammation. Crit Rev Immunol. 2015;35:401-15.

53. Theuns J, Van Broeckhoven C. Transcriptional regulation of Alzheimer's disease genes: implications for susceptibility. Hum Mol Genet. 2000;9:2383-94.

54. Liu G, Park YJ, Tsuruta Y, Lorne E, Abraham E. p53 attenuates lipopolysaccharide-induced NF-kappaB activation and acute lung injury. J Immunol. 2009;182:5063-71.

55. Banks WA, Robinson SM. Minimal penetration of lipopolysaccharide across the murine blood-brain barrier. Brain Behav Immun. 2010;24:102-9.

56. Pan W, Kastin AJ. TNFalpha transport across the blood-brain barrier is abolished in receptor knockout mice. Exp Neurol. 2002;174:193-200.

57. Lambert JC, Ibrahim-Verbaas CA, Harold D, Naj AC, Sims R, Bellenguez C, DeStafano AL, Bis JC, Beecham GW, Grenier-Boley B, et al. Meta-analysis of 74,046 individuals identifies 11 new susceptibility loci for Alzheimer's disease. Nat Genet. 2013;45:1452-8.

58. Keilani S, Lun Y, Stevens AC, Williams HN, Sjoberg ER, Khanna R, Valenzano $\mathrm{KJ}$, Checler F, Buxbaum JD, Yanagisawa $\mathrm{K}$, et al. Lysosomal dysfunction in a mouse model of Sandhoff disease leads to accumulation of gangliosidebound amyloid-beta peptide. J Neurosci. 2012:32:5223-36.

59. Conzelmann E, Sandhoff K. AB variant of infantile GM2 gangliosidosis: deficiency of a factor necessary for stimulation of hexosaminidase Acatalyzed degradation of ganglioside GM2 and glycolipid GA2. Proc Natl Acad Sci U S A. 1978;75:3979-83.

60. Cho MH, Cho K, Kang HJ, Jeon EY, Kim HS, Kwon HJ, Kim HM, Kim DH, Yoon SY. Autophagy in microglia degrades extracellular beta-amyloid fibrils and regulates the NLRP3 inflammasome. Autophagy. 2014;10:1761-75.

61. Plaza-Zabala A, Sierra-Torre V, Sierra A. Autophagy and microglia: novel Partners in Neurodegeneration and Aging. Int J Mol Sci. 2017;18(3). https:// doi.org/10.3390/ijms18030598

62. Kang J, Rivest S. Lipid metabolism and neuroinflammation in Alzheimer's disease: a role for liver $X$ receptors. Endocr Rev. 2012;33:715-46.
63. Sasaki Y, Hoshi M, Akazawa C, Nakamura Y, Tsuzuki H, Inoue K, Kohsaka S. Selective expression of Gi/o-coupled ATP receptor P2Y12 in microglia in rat brain. Glia. 2003:44:242-50.

64. Satoh J, Kino Y, Asahina N, Takitani M, Miyoshi J, Ishida T, Saito Y. TMEM119 marks a subset of microglia in the human brain. Neuropathology. 2016;36:39-49.

65. Safaiyan S, Kannaiyan N, Snaidero N, Brioschi S, Biber K, Yona S, Edinger AL, Jung S, Rossner MJ, Simons M. Age-related myelin degradation burdens the clearance function of microglia during aging. Nat Neurosci. 2016;19:995-8.

66. Wisniewski JR, Hein MY, Cox J, Mann M. A "proteomic ruler" for protein copy number and concentration estimation without spike-in standards. Mol Cell Proteomics. 2014;13:3497-506.

\section{Ready to submit your research? Choose BMC and benefit from:}

- fast, convenient online submission

- thorough peer review by experienced researchers in your field

- rapid publication on acceptance

- support for research data, including large and complex data types

- gold Open Access which fosters wider collaboration and increased citations

- maximum visibility for your research: over $100 \mathrm{M}$ website views per year

At BMC, research is always in progress.

Learn more biomedcentral.com/submissions 